San Fernando Valley state College

\title{
A COMPARISON OF SEVERAL MEASURES OF INVARIANCE
}

\begin{abstract}
A thesis submitted in partial satisfaction of the requirements for the degree of Master of Arts in

Psychology

by
\end{abstract}

Ansön Justin Levine

July, 1967 
The thesis of Anson Justin Levine is approved:

San Fernando Valley State College

July, 1967 


\section{Acknowledgments}

I should like to acknowledge the efforts of Bryce $C$. Schurr who, despite many other burdens, generously gave his own time to help me expedite the data processing.

Processing of the data could not have been accomplished without the invaluable assistance and facilities provided by Western Data Processing Center, University of California, Los Angeles.

Finally; I wish to express my appreciation and gratitude to my wife who worked very hard to make this paper possible. 


\section{Table of Contents}

Page

Acknowledgments . . . . . . . . . . . .

Abstract . . . . . . . . . . . .

viii

Introduction . . . . . . . . . .

Method . . . . . . . . . . .

Results .. . . . . . . . . . . .

Discussion . . . . . . . . . . . . .

Conclusion . . . . . . . . . . . . . •

References . . . . . . . . • • • •

Appendix . . . . . . . . . . . . 
LIST OF TABLES

1. Identical Scores and Procedures

Modified Coefficient of Invariance . . . .

2. Identical Scores and Rotation

Coefficient of Congruence

Coefficient of Invariance . . . . . . . .

3. Identical Scores and Rotation

Modified Coefficient of Invariance . . . .

4. Original Scores and Permuted Scores

30\% Rows

Coefficient of Congruence . . . . . . .

5. Original scores and Permuted scores

$30 \%$ Rows

Modified Coefficient of Invariance . . . .

6. Original Scores and Permuted scores

$60 \%$ Rows

Coefficient of Congruence . . . . . . .

7. Original Scores and Permuted Scores

90\% Rows

Coefficient of Congruence . . . . . . .

8. Original Scores and Permuted Scores

30 응 Columns

Coefficient of Invariance (Z) . . . . . .

9. Original Scores and Permuted Scores

$30 \%$ Columns

Coefficient of Invariance $\left(Z^{*}\right)$. . . . .

10. Original Scores and Permuted Scores

$30 \%$ Columns

Modified Coefficient of Invariance . . . .

11. Original Scores and Permuted Scores

$60 \%$ Columns

Coefficient of Invariance (z) . . . . . . .

12. Original Scores and Permuted scores

60\% Columns

Coefficient of Invariance $\left(Z^{*}\right)$........ 
13. Original Scores and Permuted Scores 90\% Columns

Coefficient of Invariance (z) . . . . .

14. Original Scores and Permuted Scores $90 \%$ Columns Coefficient of Invariance $\left(Z^{*}\right)$. . . . .

15. Original Scores and Permuted Scores $30 \%$ Rows and Columns Coefficient of Congruence . . . . . . .

16. Original Scores and Permuted Scores $30 \%$ Rows and Columns

Coefficient of Invariance (z) . . . . .

17. Original scores and Permuted Scores $30 \%$ Rows and Columns

Coefficient of Invariance $\left(Z^{*}\right)$. . . . .

18. Original Scores and Permuted Scores

$30 \%$ Rows and Columns

Modified Coefficient of Invariance . . . .

19. Original Scores and Permuted Scores

$60 \%$ Rows and Columns

Coefficient of Congruence . . . . . . .

20. Original Scores and Permuted Scores

$60 \%$ Rows and Columns

Coefficient of Invariance (z) . . . . . .

21. Original Scores and Permuted Scores

$60 \%$ Rows and Columns

Coefficient of Invariance $\left(Z^{*}\right)$. . . ...

22. Original Scores and Permuted Scores

100\% Rows and Columns

Coefficient of Congruence . . . . . . . .

23. Original Scores and Permuted Scores

$100 \%$ Rows and Columns

Coefficient of Invariance (z) . . . . . .

24. Original Scores and Permuted scores

$100 \%$ Rows and Columns

Coefficient of Invariance $\left(z^{*}\right)$. . . . . 
25. Original Scores and Permuted Scores $100 \%$ Rows and Columns

Modified Coefficient of Invariance . . . .

26. Original (Pre) Scores and Post Scores

Typical study

Coefficient of Congruence

Based on Principal Components . . . . . .

27. Original (Pre) Scores and Post Scores

Typical study.

Coefficient of Invariance (z)

Based on Principal Components . . . . . .

28. Original (Pre) Scores and Post Scores

Typical study

Coefficient of Invariance $\left(\mathrm{Z}^{*}\right)$

Based on Principal Components . . . . . .

29. Original (Pre) Scores and Post Scores

Typical study

Modified Coefficient of Invariance

Based on Varimax Loadings . . . . . . .

30. Original (Pre) Scores and Post Scores

Typical study

Coefficient of Congruence

Based on Varimax Loadings . . . . . . .

31. A Comparison of Several Measures

of Invariance

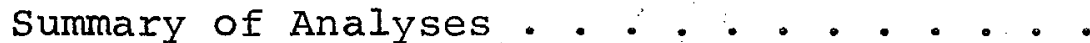

32. "Varimax Solution for Eight Physical

variables" . . . . . • . . . • • •. 
ABSTRACT

A COMPARISON OF S SEVERAI MEASURES

OF INVARIANCE

by

Anson Justin Levine

Master of Arts in Psychology

Julỳ, 1967

Criticisms have been raised against factor analysis for lack of objective and reliable methods for comparing sets of factor loadings. In an attempt to meet such criticisms, three measures of invariance or similarity of factors have been offered: the coefficient of congruence, the coefficient of invariance and the modified coefficient of invariance.

The present study develops criteria for an adequate measure of invariance and compares each of the measures with these criteria. The five criteria presented are based on considerations of what value a good measure of invariance should yield when applied to five transformations of an initial data matrix: (1) Identical scores and procedures, (2) Identical scores and rotation, (3) Row permutation, (4) Column permutation, and (5) Row and 
column permutation.

The results showed that the coefficient of congruence did not meet the specified criteria for an adequate measure of invariance for any of the cases involving permuted data matrices. The coefficient of invariance met these. criteria; however, it did not produce a unique solution. The modified coefficient of invariance did not meet the originally'specified criteria in those cases involving identical scores and varimax rotation, or row permutation. When it was discovered that the varimax factors were not orthogonal, alternative criteria for an adequate measure of invariance were developed. These new criteria reveal that the modified coefficient of invariance is a more adequate measure of the similarity of sets of factors than either of the other measures. Further results are presented for the modified coefficient and the other measures, for a typical (pre-post) application of measuring the invariance of factors. 


\section{Introduction}

One of the most.important requirements of the scientific method is that research findings can be replicated. That is, results should be achieved in such a way that another investigator repeating the exact procedures followed in the original investigation can arrive at comparable results on another occasion.

The replicability requirement has been exceedingly. difficult to meet in studies employing factor analytic techniques because objective measures of "invariance" have not been available; i.e., measures of the extent to which one has obtained comparable findings, (Harman, 1960; Henrysson, 1957; Thurstone, 1947). Thus, although the same procedures could be followed in two studies, it was not possible to objectively compare the results.

For example, one might be interested in comparing results obtained at several different colleges at which the same standard entrance examinations (e.g., SAT, SCAT, ACT) are administered. Suppose that each college, employing the same factor analytic procedures, independently analyzed its data into ten "composite tests" or factors. The problem of invariance is how similar are the factors or clusters obtained at the different colleges.

Thomson (1951) examines the reasons for employing 
factor analysis and indicates that perhaps the most fundamental advantage of the procedure is the independent parsimonious factor structure it provides to interpret or describe data. To illustrate his point Thomson provides an example of a man who can exchange five cows for so many sheep, so much cloth, a plow, etc.; however, the man soon discovers that such exchanges become much simpler if the transactions are conducted for dollars and cents instead of merchandise. Thus money can be considered a "factor" in this example. Clearly, it would not be possible to make use of the "money factor" if money did not have a consistent value from one transaction to the next. With respect to psychological investigation, one might infer that those factors with consistent, reliable, invariant interpretations are likely to be the most useful in understanding and describing behavior.

Until recently, objective methods for comparing factors have not been available. In the absence of an objective measure, investigators have had to rely on "inspection" to compare factors. As a consequence, factor analysis has been repeatedly criticized for lacking consistency and objectivity. Certainly, results achieved by the method of "inspection" are difficult, if not impossible, to repeat. Thurstone (1947) states, "... if any particular method of factoring is specified in sufficient detail, then two authors would generally get the same numerical values in the factor matrix, but that 
sort of computing agreement is not of theoretical or scientific sịgnificance (p. 363)." Recently, several methods have been suggested which appear to provide a measure of similarity of factors which is of theoretical and scientific significance.

These methods have been presented in a theoretical or strictly methodological framework without the necessary empirical results of their application to actual data, (Wrigley and Neuhaus, 1955; Pinneau and Newhouse, 1964; and Pinneau, Schurr, and Levine, 1966). In some cases authors have presented new methods of measuring invariance and accompanied these presentations only with a practical example of their method, while others chose to present hypothetical examples demonstrating the deficiencies of other measures in comparison to their own. In any case, the investigator who is faced with the problem of measuring the degree of relationship between two sets of factors has no reference which contrasts the several methods of measuring invariance with actual criteria of what an adequate measure should yield.

The intention of the present study is to develop criteria for an adequate measure of invariance and to determine the extent to which a number of different methods of measuring invariance meet these criteria.

The methods which will be compared consist of the coefficient of congruence, Wrigley and Neuhaus (1955), coefficient of invariance, Pinneau and Newhouse (1964), and 
the modified coefficient of invariance, Pinneau, schurr, and Levine (1966).

There are a number of designs to which a measure of invariance may be applied. Under one design the subjects of the two studies are fixed but the variables are different. A second design may involve different subjects but fixed variables. Still another design may involve fixed subjects and fixed variables on two occasions. The term "fixed" is used, as defined by Pinneau, Schurr, and Levine (1966), "... to imply constant subjects or variables, listed in the same order for both sets of data (p. 2)." Generally, in any of the above designs, the term "invariance" refers to the relationship between the factors of the two studies. However, Pinneau and Newhouse (1964), show that the conditions which are necessary to fulfill the requirements of the coefficient of congruence are different from those required for the coefficient of invariance in all but the fixed-variable, fixed-subject design. Consequently, the measures can be compared only for this case. 


\section{Method}

In order to adequately compare the methods of measuring invariance for each of the conditions, eleven separate correlation matrices were factor analyzed according to the principal components solution developed in Harman (1960). Each principal components solution was rotated according to the varimax criteria, Kaiser (1958). In these computations the methods remained identical for each analysis. The diagonal elements of each correlation matrix were set equal to 1.0 and the criterion employed to stop extracting additional factors was that of lambda $(\lambda)$ less than 1.0 .

Before the three measures of invariance are defined, the basic equations of principal components analysis are presented. In these equations the initial correlation matrix $\mathrm{R}$ represents the degree of relationship between the original scores which have been standardized (z), i.e., the mean and standard deviation of each column of the matrix of original scores is made zero and one, respectively. The equations which follow represent two sets of data factor analyzed using the procedure of principal components; in both instances all of the factors necessary to reproduce the correlation matrix are retained.

Where $I$ and $I^{*}$ are matrices of factor loadings and 
$S$ and $S^{*}$ are matrices of factor scores,

$\frac{1}{N} Z^{\prime} Z=R=L \quad L^{\prime} \quad \frac{I}{N} Z^{*} Z^{*}=R^{*}=L^{*} L^{*}$

$Z=S L^{\prime} \cdot Z^{*}=S^{*} L^{*}$

$S=Z L^{-I^{\prime}} \quad S^{*}=Z^{*} L^{*-I^{\prime}}$.

Figure 1 presents the above relationships. The measures of invariance. may now be defined for the case of fixed variables and fixed subjects.

As noted earlier, the method of inspection involves a visual comparison of the loading of each variable on each "composite variable". or factor in one study with the weighting of each variable on the factors of the aecond study. Since this procedure is highly contaminated by the biases and expectations of the investigator, it cannot be regarded as a scientific method. Hence, the present paper will only deal with the three objective measures of invariance.

$\therefore$ Three Objective Methods of Measuring Invariance

Coefficient of Congruence

- The coefficient of congruence may be considered as a matrix of correlations, $\mathrm{R}_{\mathrm{C}}$ ' between two sets of factor scores, $S$ and $S^{*}$ :

$$
R_{C}=\frac{I}{N} S^{\prime} S^{*} \text {. }
$$

Since $S=z L^{-1}$ and $S^{*}=Z^{*} L^{*}{ }^{-1}$, by substitution 

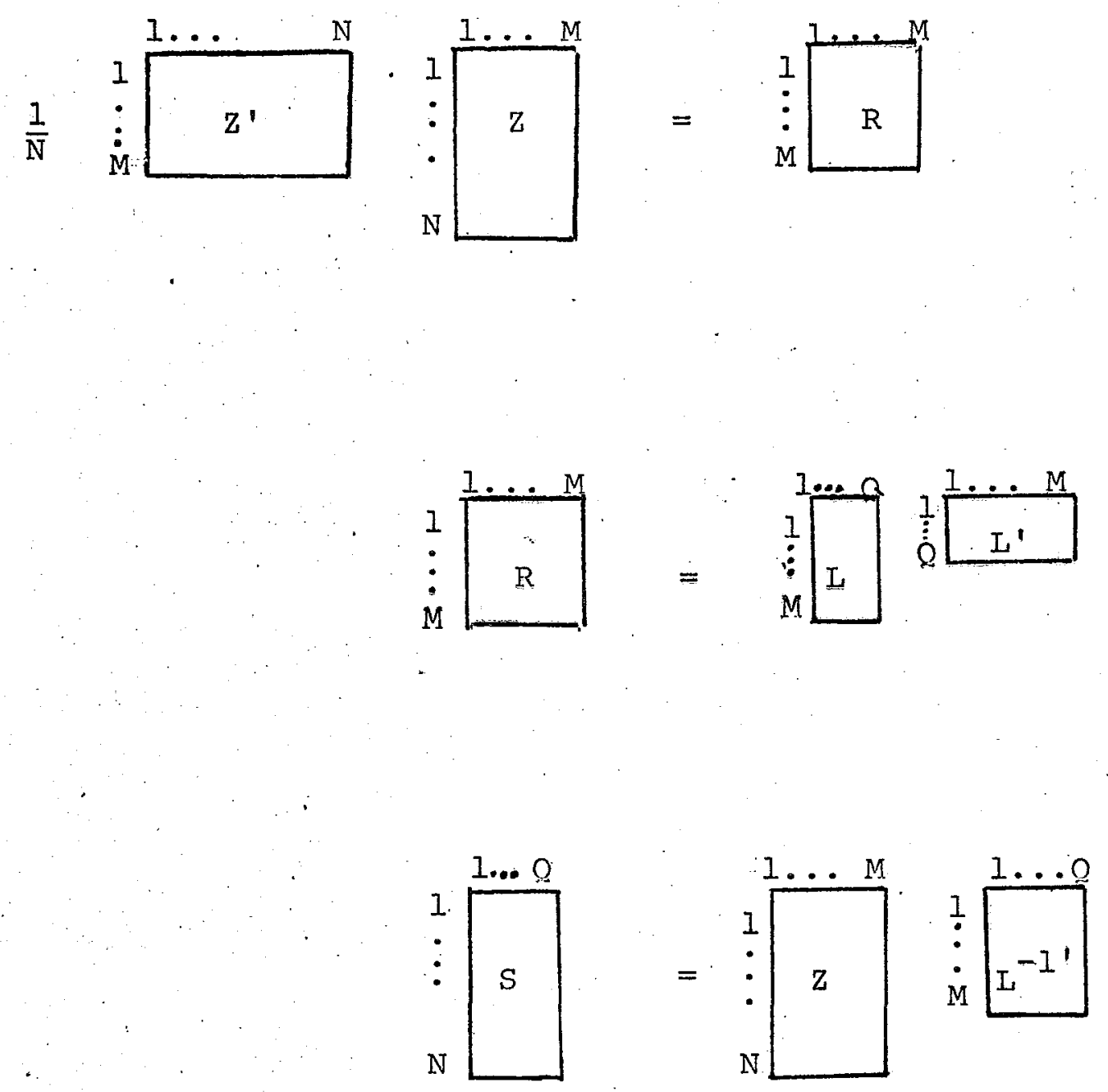

FIG. 1. Equations for Factor Loadings and Factor Scores 
the coefficient of congruence becomes

$$
R_{C}=\frac{1}{\bar{N}} L^{-1} \quad Z^{\prime} Z^{*} L^{*-1}
$$

The matrix of intercorrelations between the different $z$ matrices is given by the equation

$$
\frac{\mathrm{I}}{\mathrm{N}} \mathrm{Z}^{\prime} \mathrm{Z}^{*}=\mathrm{R}_{12}
$$

hence, by substitution,

[2]

$$
R_{C}=L^{-1} \quad R_{12} \quad L^{*-1}
$$

Coefficient of Invariance

The coefficient of invariance, $R_{I V}$ is the matrix of correlations between two sets of factor scores, $S$ and $S_{Z}^{*}$, where

$$
\begin{aligned}
& S=Z L^{-I^{\prime}} \text { and } S_{Z}^{*}=Z L^{*-I^{\prime}} ; \text { thus } \\
& \mathrm{R}_{I V_{Z}}=\frac{I}{\bar{N}} S^{\prime} S_{Z}^{*} \\
& =\frac{1}{N} L^{-1} Z^{\prime} \quad z \quad L^{*-1} \text {. }
\end{aligned}
$$

The intracorrelation matrix for the first set of variables, $R_{I I}=\frac{1}{N} Z^{\prime} \mathrm{Z}$, is a factor in this equation; hence, by substitution

$$
\begin{aligned}
R_{I V_{Z}} & =I^{-1} R_{I I} L^{*-I^{\prime}} \\
& =L^{-1} \quad \text { L } L^{\prime} I^{*-I^{\prime}} \\
& =I L^{\prime} L^{*-I^{\prime}}
\end{aligned}
$$




$$
=L^{\prime} L^{*-1} \text {. }
$$

Alternatively, the coefficient of invariance, $\mathrm{R}_{I V_{Z} *^{\prime}}$ may be computed using factor scores based upon $\mathrm{Z}^{*}$.

If $S_{Z^{*}}=Z^{*} I^{-I^{\prime}}$ and $S^{*}=Z^{*} I^{*-I^{\prime}}$,

$$
\begin{aligned}
\mathrm{R}_{I V_{Z^{*}}} & =\frac{1}{N} S_{Z^{*}}^{\prime} S^{*} \\
& =\frac{1}{N} L^{-1} \quad Z^{* \prime} \quad Z^{*} \quad L^{*-I^{\prime}} .
\end{aligned}
$$

The intracorrelation matrix among the second set of variables, $R_{22}=\frac{1}{N} Z^{*}, Z^{*}$, appears as a factor in this equation; hence,

$$
\begin{aligned}
R_{I V_{Z^{*}}} & =I^{-1} R_{22} \mathrm{~L}^{*-1} \\
& =I^{-1} \mathrm{~L}^{*} \mathrm{~L}^{*} \mathrm{~L}^{*-1} \\
& =I^{-1} \mathrm{~L}^{*} \mathrm{I} \\
{[4] } & =\mathrm{I}^{-1} \mathrm{~L}^{*}
\end{aligned}
$$

Modified Coefficient of Invariance

The modified coefficient of invariance, Ro ${ }^{\prime}$, is also the correlation between two sets of factor scores, so and $S * 0$ :

$$
R_{I V}=\frac{I}{N} \text { so' } S * 0,
$$

In this case the matrix of factor scores is computed using a matrix of orthogonal standard scores $\left(z^{\circ}\right)$, i.e., the 
subjects' scores on one variable are independent of their scores on each of the other variables. The two sets of factor scores, $\mathrm{S}^{\circ}$ and $\mathrm{S} * 0$, are developed using the same $\mathrm{Z}^{\circ}$ matrix and two different sets of factor loadings:

and

$$
\begin{aligned}
& S^{\circ}=Z^{\circ} L^{-I} \\
& S^{\circ}{ }^{\circ}=Z^{\circ} L^{-1}{ }^{\prime} \because
\end{aligned}
$$

By substitution, the equation for the modified coefficient of invariance becomes:

$$
\mathrm{R}_{I V}^{O}=\frac{1}{\bar{N}} \mathrm{~L}^{-1} \mathrm{Z}^{\prime} \text { Z } \mathrm{Z}^{\circ} \mathrm{L}^{*-I^{\prime}} .
$$

In this equation, $\frac{1}{N} z^{\circ} z^{\circ}=R_{I}=I$, since the variables are perfectly correlated with themselves and uncorrelated with each others hence,

$$
\begin{aligned}
R_{I V}^{0} & =I^{-1} \quad I \quad I^{*-1} \cdot \\
& =I^{-1} \quad I^{*-1} \cdot
\end{aligned}
$$

The three methods that have been proposed to measure invariance all center around the issue of how similar the weights or contributions of the variables to each factor of one study are to the loadings or weights of the same variables on each factor of another study. Ideally, each of these methods of measuring invariance represents an attempt to quantify the degree of relationship between the two sets of factor loadings. In each instance the procedure may be viewed as the correlation between factor scores based on the two sets of loadings, one from each' 
study; hence, the letter " $R$ " is used in the designation of all three measures. Conceived in this manner, the differences between the measures involve the method used to compute the factor scores.

A letter subscript is used to differentiate the three measures and to indicate that the relationships are actually correlations between two sets of factor scores rather than two sets of standard scores. However, the three measures of invariance yield different results under certain conditions because the factor scores they employ are based on different $z$ matrices. Deciding which $z$ matrices are to be used becomes a decision as to which of the three measures most gatiofactorily meet criteria of an adequate measure of invariance.

\section{Five Conditions for Measuring Invariance}

The following conditions have been developed in order to establish criteria for an adequate measure of invariance. These conditions actually represent data matrices which are used to compute the appropriate factor scores for each of the three objective measures of invariance. These five sets of data represent purely artificial circumstances in which the nature of the relationships. can be predicted a priori. These data are important because they represent a condition where criteria for a good measure of invariance may be specified before the invariance of the two sets of factors are computed. Thus, it will be possible 
to state the desired result with each case and to see which measure most satisfactorily meets the specified criteria.

Rather than focus on mathematical relationships between the various measures, the present research tests the measures using actual data. The advantage of employing actual data rests upon the expectation that the results may reveal additional relationships which lead to increased comprehension of the advantages and disadvantages inherent in employing one or another of the measures of invariance.

Criterion 1: Identical Scores and Procedures

Two sets of identical scores yield the same factors; thus, an adequate measure of invariance should show that matching factors of the two sets are perfectly related.

In this case of identical scores and procedures, each score of each individual on the variables in the pre test are identical with the scores of each individual on the same set of variables on the post test. The factor analytic procedures used are identical for both studies. In order to accomplish this comparison, instead of using. the actual post test scores, a duplicate set of pre scores are substituted for the post scores so that the comparison is between identical factor scores based on identical sets of data. When $\mathrm{Z}=\mathrm{Z}^{*}$, I should equal $\mathrm{L}^{*}$.

The value of this example rests on the fact that two sets of identical scores should yield identical sets of factor loadings if the methods of determining the factors 
are the same for each set. Thus, an appropriate invariance measure should show a perfect matching. Since the measures being considered are correlation coefficients, each should yield a value of 1.00 for matching factors.

Criterion 2: Identical Scores and Rotation

One may obtain a second set of factor loadings $L$ * by post multiplying the principal components loading matrix by an orthonormal transformation matrix $\mathrm{T}$; $i . e$. , $L^{*}=\mathrm{I}$ T. Therefore, an adequate measure of invariance should indicate that $I *$ is just an orthonormal transformation of $I$.

In the present study the three measures of invariance utilize the principal components factors and the same factors rotated by the varimax method developed by Kaiser (1958): The varimax method utilizes an iterative procedure to obtain the orthonormal transformation of the row normalized loadings. The scores on the variables are not modified in any way; $i . e$. , the standard score matrices in both pre and post (rotation) test are still equal.

\section{Criterion 3 : Permuted Rows}

An adequate measure of invariance should show that two sets of factor loadings are perfectly related when the factor loadings are identical. Such conditions are offered by Pinneau and Newhouse (1960) as evidence of the superiority of their measures over those presently available. The following development demonstrates that permuting rows 
does not modify the correlation matrix, and hence does not modify the factor loadings. If $P$ is the permutation $\operatorname{matrix}, \mathrm{Z}^{*}=\mathrm{P} \quad \mathrm{Z}$,

$$
R^{*}=\frac{1}{N} Z^{* 1} Z^{*}
$$

and, by substitution,

$$
\begin{aligned}
R^{*} & =\frac{1}{\bar{N}}\left[\begin{array}{ll}
P & Z
\end{array}\right]^{\prime} \cdot P \quad Z \\
& =\frac{1}{N} \cdot Z^{\prime} \quad P^{\prime} \quad P \quad Z \\
& =\frac{1}{N} Z^{\prime} I \quad Z \\
& =\frac{1}{N} Z^{\prime} Z \\
& =R .
\end{aligned}
$$

If the rame procedures are enployed to factor the correlation matrices produced by $\mathrm{z}$ and $\mathrm{Z}^{*}$, and these correlation matrices are identical, then the two sets of loadings should also be identical.

Three cases of row permutation are presented; for each of these cases a different number of rows are interchanged. This procedure is followed in order to systematically increase the amount of change in the original matrix of standard scores. Thus, it is possible to ascertain the extent to which these modifications effect the different measures of invariance. Ideally, the three measures should continue to yield the same coefficients if the sets of factor loadings remain identical.

In the first case $30 \%$ of the 274 rows of the original pre test matrix of standard scores $(Z)$ are interchanged 
randomly. The resulting matrix $Z^{*}$ is a row permutation of the original set of standard scores. In the second case $60 \%$ of the rows of $\mathrm{Z}$ are randomly interchanged. Finally, $90 \%$ of the rows of $\mathrm{Z}$ are permuted. These three cases should indicate what happens to the measures of invariance when the relationships between the rows of the permuted matrix become more and more disparate.

Since'the relationship between the variables is not disturbed by permuting the rows of the data matrix, the intracorrelation matrix for each of the three permuted matrices should be the same as that obtained for $\mathrm{z}$. As stated by Pinneau, Schurr and Levine (1966), an appropriate measure of invariance should reflect the degree of correspondence between the two sets of factor loadings. Thus the criteria for an adequate measure of invariance in this case is an identity matrix.

Criterion 4: Permuted Columns

Disturbing the order of the scores on the variables, i.e., the columns of $z$, results in two sets of factor loadings which are not identical. Therefore an adequate measure of invariance should show that changing the order of the columns modifies the relationships between the two sets of factors.

Instead of interchanging a row of $z$ to form $z$, in this case all of the scores for one variable or column are randomly reassigned to new column locations in $\mathrm{Z}^{*}$. The 
relationship between the columns of the original matrix and the permuted column matrices are gradually reduced by randomly interchanging $30 \%$ of the original columns (14 columns), $60 \%$ of the original columns (28 columns), and $90 \%$ of the original columns (4I columns). For this case,

$$
\begin{aligned}
& \mathrm{Z}^{*}=\mathrm{Z} \mathrm{P} \\
& \mathrm{R}^{*}=\frac{\mathrm{I}}{\mathrm{N}} \mathrm{Z}^{* 1} \quad \mathrm{Z}^{*} ;
\end{aligned}
$$

by substitution

$$
\begin{aligned}
& \mathrm{R}^{*}=\frac{1}{\mathrm{~N}}\left[\begin{array}{ll}
\mathrm{Z} & \mathrm{P}
\end{array}\right]^{\prime} \quad \mathrm{Z} \quad \mathrm{P} \\
& =\frac{1}{N} P^{\prime} \quad Z^{\prime} \quad Z \quad P \\
& =P^{\prime} R \quad P \text {. }
\end{aligned}
$$

Since permuting the rows and/or columns of a matrix does not effect the value of the numerical entries, only their location; the preceding equation shows that $R$ and $R^{*}$ contain the same numerical entries. The values appearing in a given row and column are the same for only those cases in which unity appears in the principal diagonal of P. Thus, the intracorrelation matrix of $z$ is not equal to that of $Z^{*}$. If $R^{*}=P^{\prime} R$ P and $P \quad L=L^{*}$ then, by substitution,

$$
\begin{aligned}
R^{*} & =P I L^{\prime} P^{\prime} \\
& =L^{*} L^{*} .
\end{aligned}
$$

Therefore, it may be seen that although the matrices of loadings contain the same entries, the loading values are attached to different variables; hence, the factors are 
no longer the same. If the loadings for the variables have changed, then the measures of invariance of the two sets of loadings should indicate that each factor has changed even if the two sets of factor scores are perfectly correlated.

Another approach,. Pinneau and Newhouse (1964), to the problem of factor similarity stresses invariance of factor scores rather than invariance of loadings. According to this view, the results of a desirable measure of invariance should yield an identity matrix between the two sets of factors (original scores versus column permuted scores). For example, if the variables of the two studies have different namer, and scorer on one of the factor ware highly related to scores on a factor of the other study, an investigator might not wish to dismiss this relationship merely because the loadings on the factor of the second study are attached to variables with different names.

\section{Criterion 5: Rows and Columns Permuted}

\footnotetext{
. The important feature of the example created by permu-

ting both rows and columns simultaneously is that as more and more rows and columns are interchanged, this case very closely approximates the circumstance in which there are only chance relationships between $\mathrm{z}$ and $\mathrm{z}^{*}$.

In a typical study within the fixed-subject, fixedvariable design, scores are collected for the same subjects on the same variables on two different occasions. In the
} 
case of permuting the rows and columns of one $z$ matrix to form $z^{*}$, again there is different data for each matrix of standard scores. In the row and column case, 30\%, 60\%, and $100 \%$ random permutations of both rows and columns should indicate the effect of the gradual distortion introduced between the original $\mathrm{z}$ :matrix and the row and column permuted matrices $\left(\mathrm{z}^{*}\right)$. In the $100 \mathrm{z}$ case, when all rows and columns have been randomly pexmuted, only by chance does $z_{i j}=z_{i j}^{*}$

If these matrices are factor analyzed, then $z=S$ I and $Z^{*}=S^{*} L^{*}$ where the relationship between $L$ and $L^{*}$ and between $S$ and $S^{*}$ is not readily evident. Indeed, as in the other cases, an adequate measure of invariance should directly reflect the comparability of $L$ and $L^{*}$.

\section{Typical Study}

The typical study represents the usual case in which pre and post data is available on the same subjects for the same variables. In such instances the numerical entries in both the post $\mathrm{z}$ matrix and the resulting factor loading matrix are different from those computed from the pre test data.

The artificial rearrangement of scores on subjects, on variables, or on subjects and variables presented in the preceding sections has a direct bearing upon the appropriate measure of invariance for the typical study. Measures of invariance which do not meet the specified 
criteria for the other cases will not satisfactorily measure the relationships between the factors in the typical study. Hence, an adequate measure of invariance should be the measure which most adequately meets the criteria established.for the previous cases. Thus, the typical study is used only to illustrate an application of each of the measures of invariance.

\section{Data Description}

The data for the analysis consists of two sets of responses (pre and post) of 274 subjects to a 46 -item attitude survey. ${ }^{1}$ The subjects were trainees who participated in a two-week Head start training program designed to prepare them to become assistant teachers or teacher aides. The attitude survey consists of 46 statements with four possible answers ("strongly agree, " "agree, " "disagree" and "strongly disagree") for each statement or item. This particular set of data was selected because of several important considerations: the size of the sample of subjects and items, the fact that such data are typically analyzed by factor analytic techniques (in fact, results of factor analyses of other adaptations of this attitude survey are available), and most important, these data

1 These data were collected by the office of Economic Opportunity Training and Development Center, Evaluations Section, and have been made available to the writer by the Director, Dr. Donald R. Thomas. 
fulfill the test-retest condition necessary to compare two sets of factors extracted from data collected on the same subjects and the same variables on different occasions. The procedure which is followed to rearrange or permute the scores of the original $\mathrm{z}$ matrix involves randomly interchanging entire rows (or columns) of $\mathrm{Z}$ without changing the original. row or column headings. This is achieved by pre (or post) multiplying the original data matrix by a random permutation matrix.

\section{Summary of Criteria}

The following criteria have been developed for an adequate measure of invariance:

1. In the case of identical scores and procedures the measure should yield an identity matrix.

2. For identical scores and rotation the result should be a transformation matrix.

3. The criterion for an adequate measure of invariance when rows are permuted is, as in the first case, an identity matrix.

4. When columns are permuted, an adequate measure should not yield an identity matrix; rather, it should reflect the increasing disparity between the two sets of factor loadings as more and more columns are rearranged.

5. Row and column permutation should indicate that the two sets of factors are not identically 
related and that the relationship between the loadings decreases as the amount of permutation increases.

The measure which satisfactorily meets the criteria of an adequate measure of invariance in the artificial cases should most directly reflect the relationship between the two sets of factor loadings in the typical study. 


\section{Results}

The following section presents results achieved for the three measures of invariance under each of the conditions described earlier. These five conditions represent artificial situations for which criteria for an adequate measure of invariance were developed prior to computing the results for each measure. In addition, for several cases results are presented which exceed the scope of the original criteria; these additional results are presented because they merit consideration in selecting an appropriate measure of the similarity or invariance between two sets of factors. Results are also presented for a typical application of the measures of invariance.

In order to distinguish the two sets of factors which are being compared, a Roman numeral without a prime will represent a factor from the first set of factors, e.g., Factor I of the pre test scores. A factor from the second set of factors is denoted by a Roman numeral and a prime, e.g., Factor III' of the post test scores.

Identical Scores and Procedures

The criterion for an adequate measure of invariance for the case of identical $z$ scores, correlation matrices, factor loadings, and factor scores was an identity matrix. 
Although differential criteria were not established with respect to unrotated versus rotated factor solutions, the results presented below indicate that additional considerations arise when two identical varimax solutions are compared.

Unrotated Factor Loadings

Comparing factor scores computed from two identical principal components factor loadings show that the coefficient of congruence, the coefficient of invariance, and the modified coefficient of invariance result in an identity matrix. Hence, for principal component factor scores, all these measures meet the criterion of an adequate measure of invariance.

Rotated Factor Loadings

Rotating the two identical principal components loadings according to the varimax criteria produced identical varimax factor loadings which were used to compute the appropriate sets of factor scores required by each of three measures of invariance.

Only two of the three measures, the coefficient of congruence and the coefficient of invariance, met the criteria established for an adequate measure of invariance; i.e., both yielded an identity matrix.

since the modified coefficient of invariance did not result in an identity matrix, the results for this measure require more detailed presentation. Table 1 presents the 
obtained matrix of coefficients. These results show unity in the principal diagonal indicating that each factor in one set is perfectly related to itself in the second set, i.e., Factor $I=$ Factor I'; Factor II = Factor II', etc. Table I also indicated that Factor $I$ is related to Factor II', III', IV', etc. Similar relationships are also found for all of the other factors. Thus, the modified coefficient of invariance indicates that the same two factors are perfectly related; and that these factors are also interrelated with the other factors. For example, the correlation between scores on Factor I and those on Factor II' is -.2909 .

This regult rajses question concerning the orthogona lity of the varimax factor loadings. If varimax factor loadings are orthogonal, then the sum of the crossproducts of factor loadings for any two factors should equal zero. Similarly, in the case of principal components the sum of the crossproducts of the loadings on any two factors should also be zero. A comparison of Factors I and II for the varimax solution shows the sum of the crossproducts to be 1.3159; the sum of the crossproducts of the same factors in the principal components solution is .00005. Thus, the varimax solution did not result in orthogonal factors. Since this result has implications for other varimax solutions, the sum of crossproducts for the eight physical variable problem presented in Harman (1960, p. 304-305). was computed. Table 32 in the appendix shows that the 
TABLE 1

IDENTICAL SCORES AND PROCEDURES

MODIFIED COEFFICIENT OF INVARIANCE

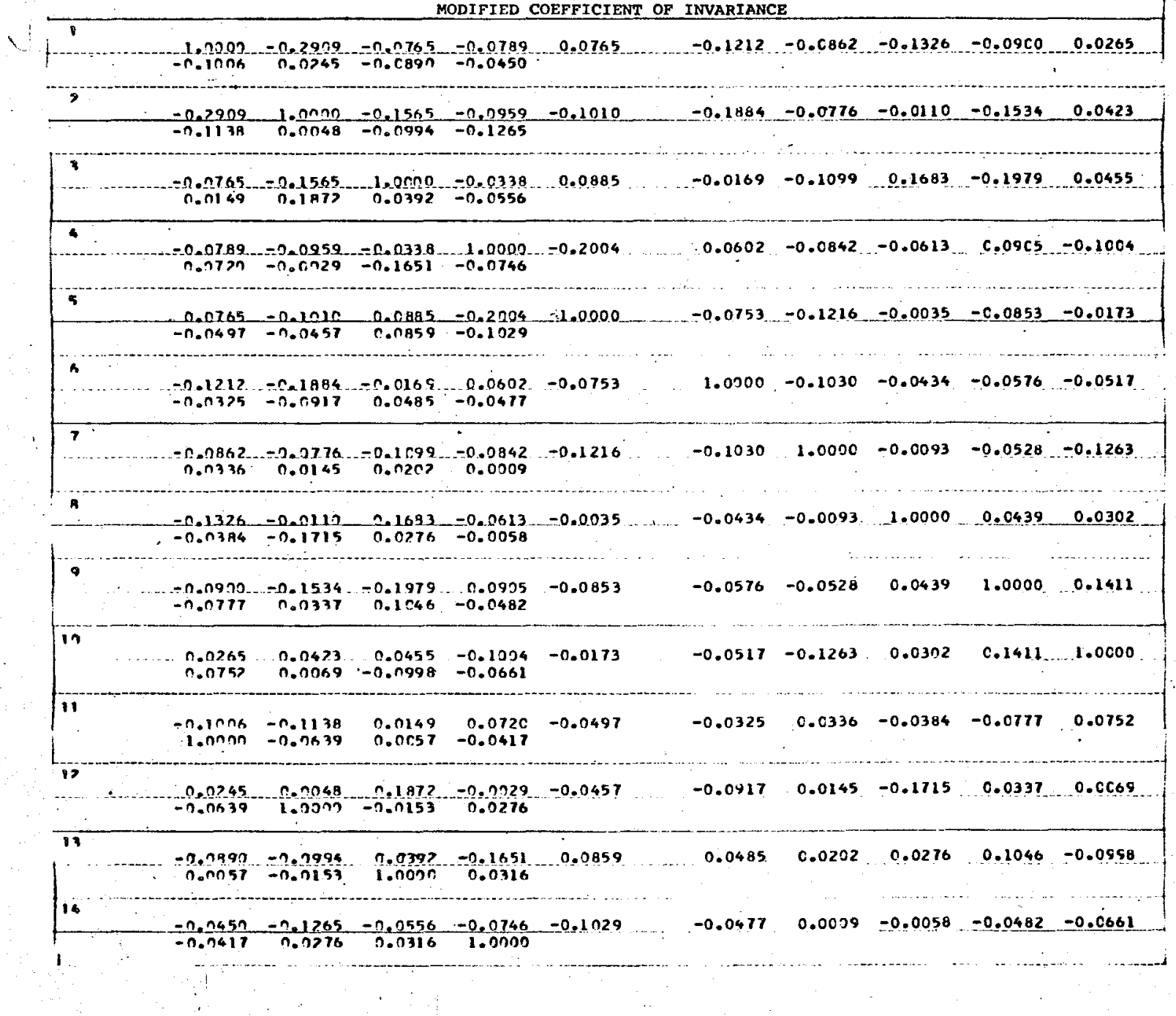


varimax solution presented by Harman also does not result in completely orthogonal factors.

\section{Identical Scores and Rotation}

The case of rotation has been presented above when both sets of factor loadings were rotated. In the present case only one of the two sets of factor scores is based upon a rotated matrix of factor loadings. Therefore, this case reflects the correspondence between one set of factor scores based on the original principal components factor loadings and the other set of factor scores based on a varimax rotation of the original principal components. The criterion established for this case is that an accurate measure of invariance should reflect the fact that the factor scores are based upon two different sets of factor loadings. Since the only change introduced into this case was that of rotating the second set of loadings, the criterion is that an adequate measure of invariance should reflect the amount the second set of loadings have been. rotated, i.e., T.

Results for Each Measure of Invariance

The coefficient of invariance and the coefficient of congruence resulted in a transformation matrix, Table 2, which will produce the varimax loadings if it is premultiplied by the original matrix of principal components factor loadings.

The modified coefficient of invariance, Table 3 , is 


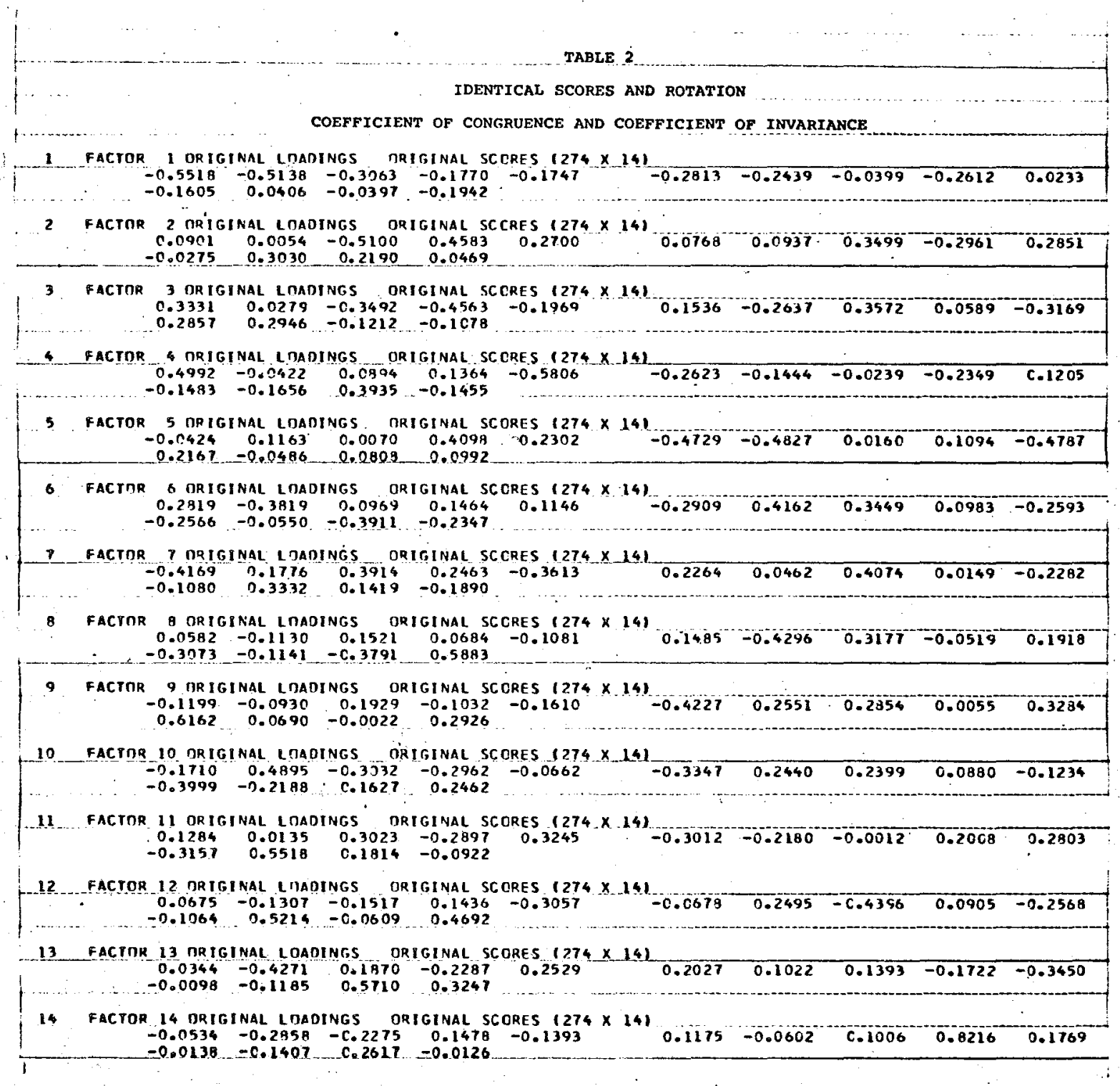


TABLE 3

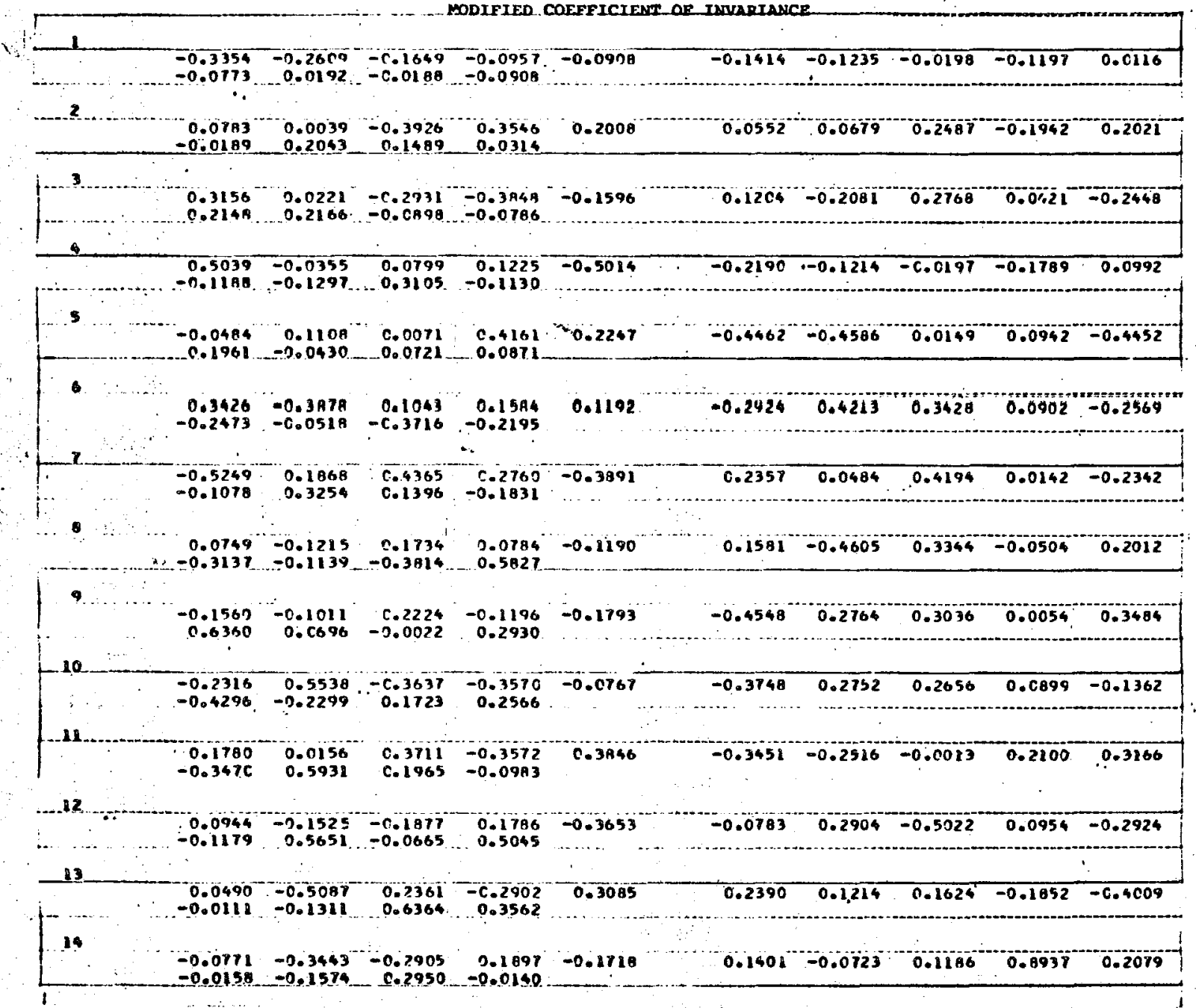


not equivalent to the transformation matrix presented for the coefficient of invariance and congruence. Indeed, the set of modified coefficients is not an orthonormal transformation matrix since the sum of squares for a row do not equal 1.00; sum of squares of unity would not be expected since the varimax loadings are not orthogonal.

\section{Permuted Rows}

The criterion established for an adequate measure of invariance in the permuted row case was that the two sets of factor loadings should be shown to be identical regardless of the number of rows permuted. This follows from the fact that despite the number of rows permuted, the correlation matrix and. the method of factoring remain identical. Therefore, an adequate measure of invariance between the original factor loadings and the factor loadings based on row permuted $z$ should yield an identity matrix.

\section{0은 Row Permutation}

Coefficient of congruence. Table 4 presents the results of correlating the appropriate factor scores computed for the coefficient of congruence. Table 4 shows that in no case did a factor of the original scores correlate 1.0 with a factor based on the $30 \%$ permuted scores; thus, this measure does not yield an identity matrix. The highest relationship measured by the coefficient of congruence was .78 for Factor XIII and XIII'. Inspection of Table 4 also shows that the relationship between the 


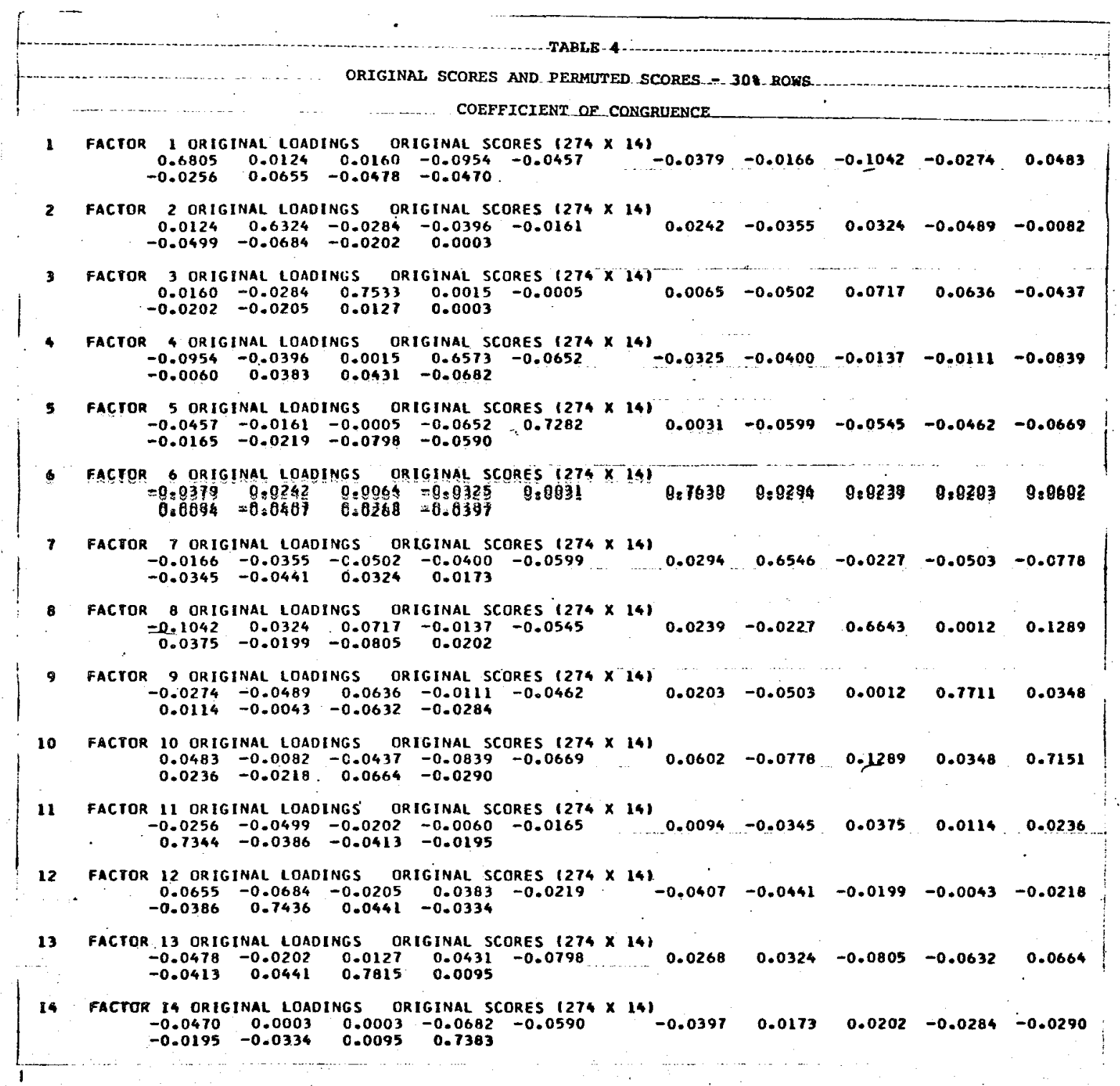


two sets of factor scores was the highest when a factor was compared with itself rather than some other factor of the second set. Thus, in the matrix of coefficients of congruence the diagonal elements show the highest relationships.

Coefficient of invariance. As mentioned above, the coefficient of invariance has two solutions, depending upon which.standard score matrix $\left(z\right.$ or $\left.z^{*}\right)$ is used to compute the factor scores. In this case both solutions satisfy the criterion established for an adequate measure of invariance.

Modified coefficient of invariance. Table 5 presents the modified coefficient of invariance for appropriate factor score matrices when $30 \%$ of the rows of the original matrix have been permuted. The results in Table 5 indicate that the modified coefficient of invariance is not an identity matrix. A comparison of Table 5 with Table 1 shows that the same values were obtained when the second set of factor scores were based on $30 \%$ row permutation $\left(z^{*}\right)$ as were obtained with identical scores and procedures based on varimax rotation.

$60 \%$ Row Permutation

Coefficient of congruence. Table 6 presents the results for the coefficient of congruence for $60 \%$ row permutation. Again, the coefficient of congruence does not produce an identity matrix; however, the principal diagonal 


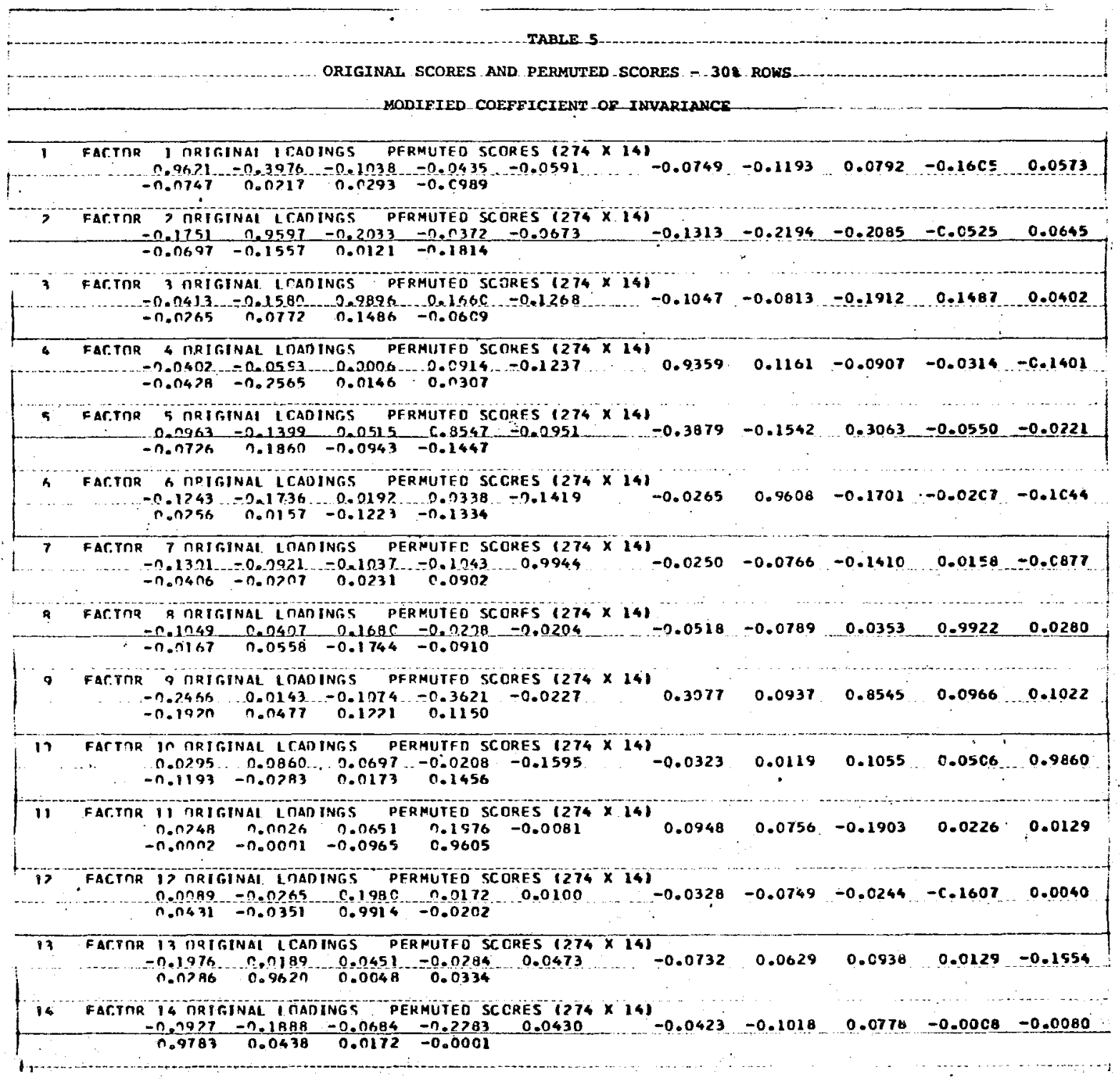




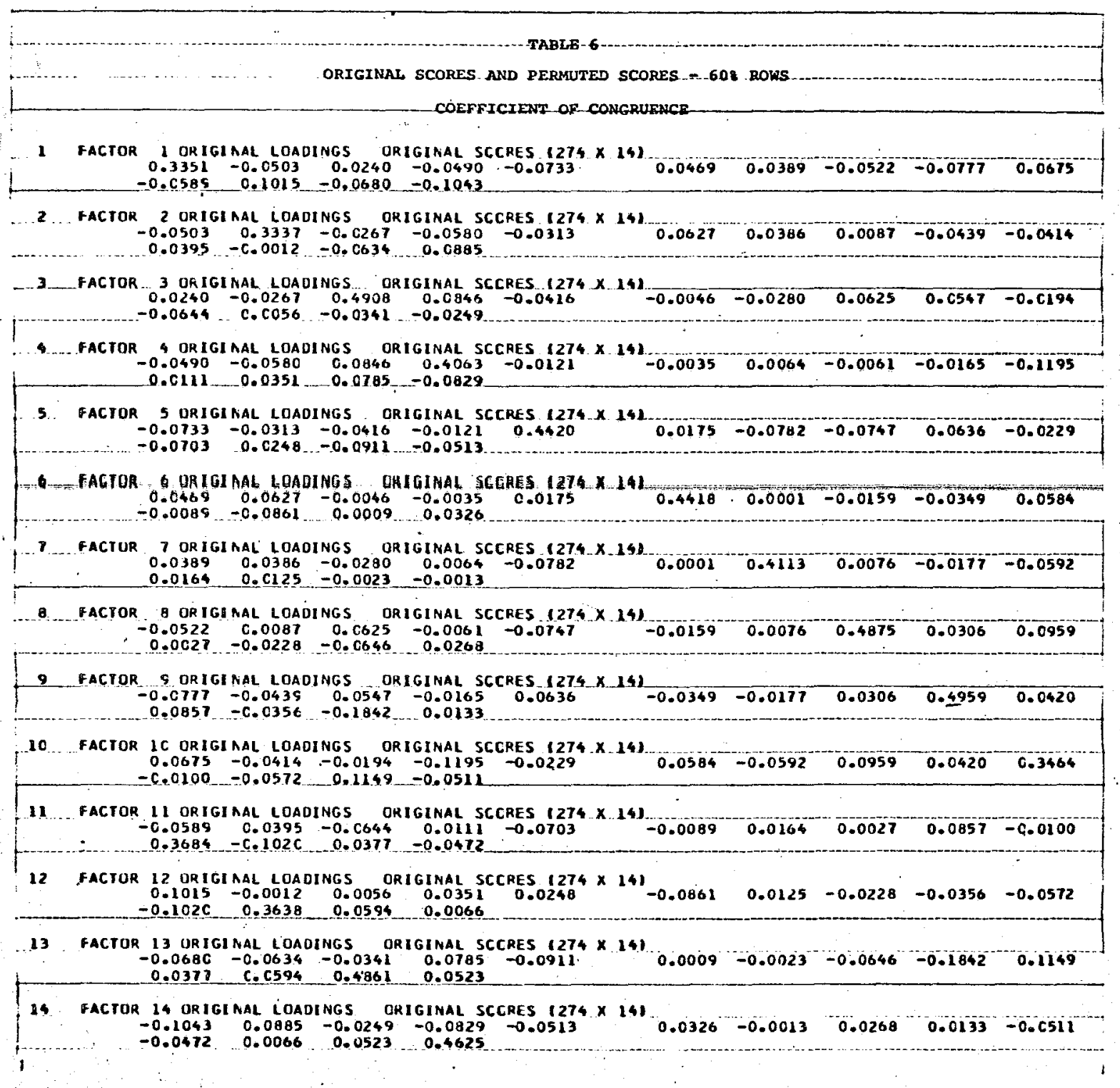


of Table 6 still contains the highest relationships. The highest of these coefficients of congruence is .4959 for the relation between Factor IX and IX'. A comparison of Tables 4 and 6 shows that the coefficients of congruence are lower when $60 \%$ of the rows of $\mathrm{z}$ are permuted than they were when $30 \%$ of the rows of $\mathrm{z}$ were permuted. The range of coefficients of congruence is .3337 to .4959 for $60 \%$ row permutation and .6324 to .7815 for $30 \%$ row permutation. Coefficient of invariance. Both solutions of the coefficient of invariance resulted in an identity matrix. Modified coefficient of invariance. The results obtained using the modified"coefficient of invariance for $60 \%$ row permutation are identical to those found for $30 \%$ row permutation, Table 5 ; hence, an additional table is not presented in this case. Thus, the modified coefficient of invariance does not yield an identity matrix for this case.

$90 \%$ Row Permutation

Coefficient of congruence. Table 7 , shows the results of the $90 \%$ row permutation case for the coefficient of congruence. As in the case of $30 \%$ and $60 \%$ row permutation, the coefficient of congruence does not produce an identity matrix.

In contrast to Tables 4 and 6 , Table 7 reveals that the highest relationships between the factors is not always in the principal diagonal. In other words, Table 7 indicates that Factor I and Factor I' are not the most 


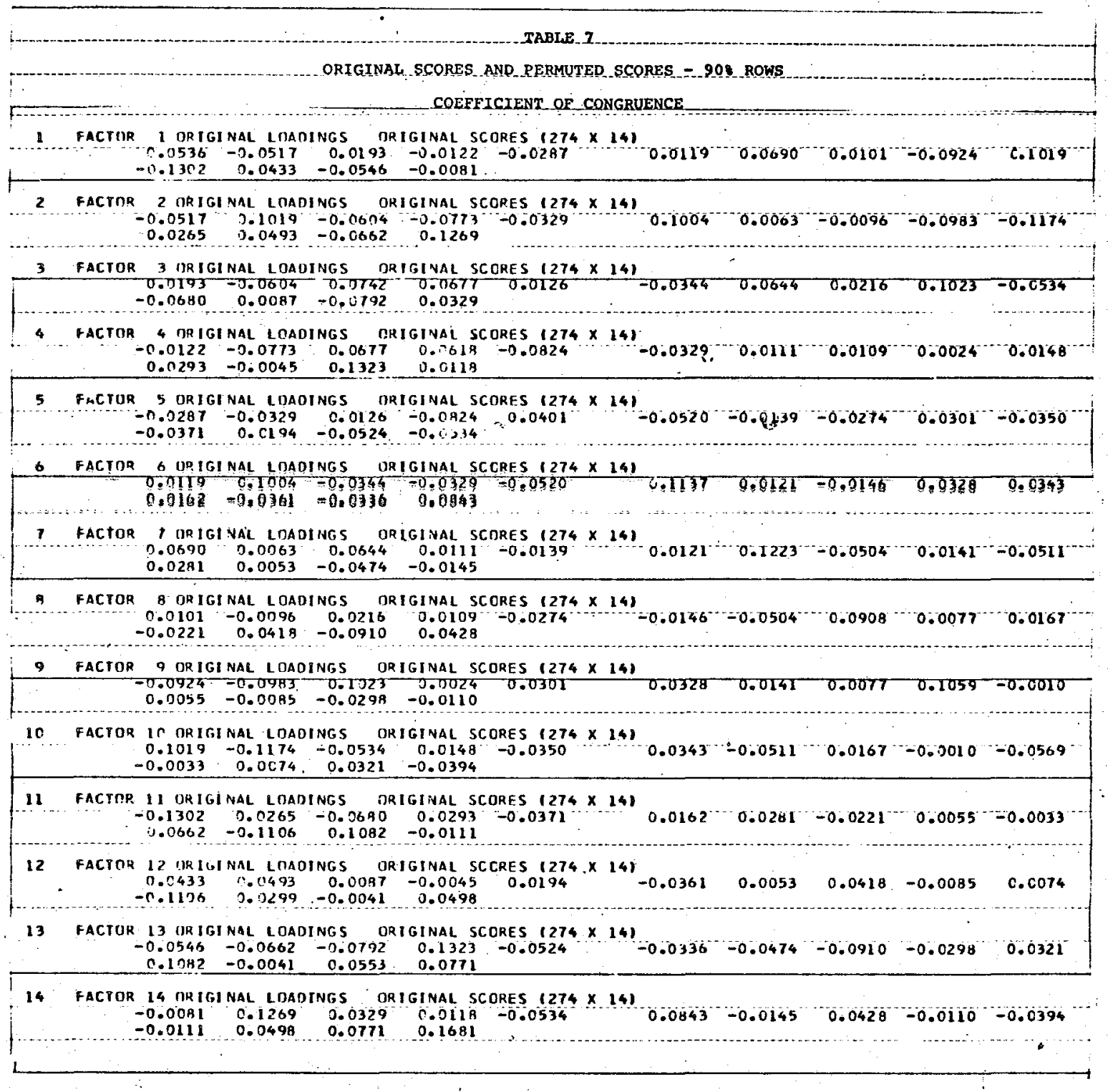


highly related even though the loadings for these two factors are identical. Factors V, VI, and XIV show that they are more highly related to themselves (i.e., to Factors $V^{\prime}, V^{\prime}$, and XIV', respectively) than to other factors of the second set. However, as in the case of Factor I, the highest relationships for the remaining ten factors of the two sets are in the off diagonal. That is, the relationships are not highest for factors of the two sets with identical loadings.

Coefficient of invariance. The results of both solutions for the coefficient of invariance were an identity matrix.

Modified coefficient of invariance. The results of the modified coefficient of invariance for this case are identical to the results presented for $30 \%$ row permutation, Table 5; hence, an additional table is not presented for this case. It will be recalled that this table is not an identity matrix.

Summary of Results for Row Permutations.

The results of permuting $30 \%, 60 \%$, and finally $90 \%$ of

the rows of $z$ to form $z^{*}$ reveal that of the three measures only the coefficient of invariance resulted in an identity matrix. The modified coefficient of invariance did not result in an identity matrix although the same results were obtained for $30 \%, 60 \%$, and $90 \%$ row permutation. The coefficient of congruence did not yield an identity matrix nor were the results the same for 
any of the cases of row permutation; as successively more rows were permuted the magnitude of the coefficients of congruence decreased. Consequently, only the coefficient of invariance showed results which completely conformed to the criterion establi.ished for an adequate measure of invariance. Both the coefficient of invariance and the modified coefficient of invariance produced results which show that permuting rows of $z$ does not change the measure of the similarity of the two sets of factors. However, the modified coefficient of invariance does not show an identity matrix because neither of the sets of factor loadings are orthogonal.

\section{Permuted Columns}

The criteria for the case of permuting columns of $z$ to form $z^{*}$ are that an adequate measure of invariance should show that the factors are not identically related; in addition, increasing the number of permuted columns should decrease the relationship between the factors. These criteria are based on the fact that permuting columns of $\mathrm{z}$ to form $\mathrm{Z}^{*}$ introduces change in the correlation matrix $\mathrm{R}^{*}$ such that $R^{*} \neq R$. Therefore, $L^{*}$ based on $R^{*}$ is not equal to $L$ based on $R$. Consequently, an adequate measure of invariance should reflect the inequality between the loadings. Further, as the number of permuted columns increases, $I$ and $L^{*}$ will become more disparate; that is, the factors based upon the original scores are becoming less related to the factors based upon the column permuted 
scores.

30\% Column Permutation

Coefficient of congruence. The result of the coefficient of congruence in this case was an identity matrix. In other words, the coefficient of congruence indicates that the two sets of factors are perfectly related when the second set is based upon the intracorrelation matrix $\mathrm{R}^{*}$ obtained from $\mathrm{Z}^{*}$, which is $\mathrm{Z}$ with $30 \%$ of its columns permuted.

Coefficients of invariance. Tables 8 and 9 present the two solutions for the coefficient of invariance. They indicate high relationships between the two sets of factors; however, the two sets of factor scores are not perfectly correlated. A closer inspection of Tables 8 and 9 indicates that one matrix of coefficients of invariance is the transpose of the other. This relationship is shown mathematically in the summary of results for the column permutation case.

Modified coefficient of invariance. The results for this measure are presented in Table 10. Examination of this table reveals that the two sets of factor scores are not perfectly related:

60\% Column Permutation

Increasing the number of permuted columns results in greater differences between the two sets of factor loadings. Consequently, an adequate measure of invariance should 
TABTE $B$

ORIGINATC SCORES AND PERMUTED SCORES = 308 COLUNAS

COEFFICIENT OF INVARIANCE (z)

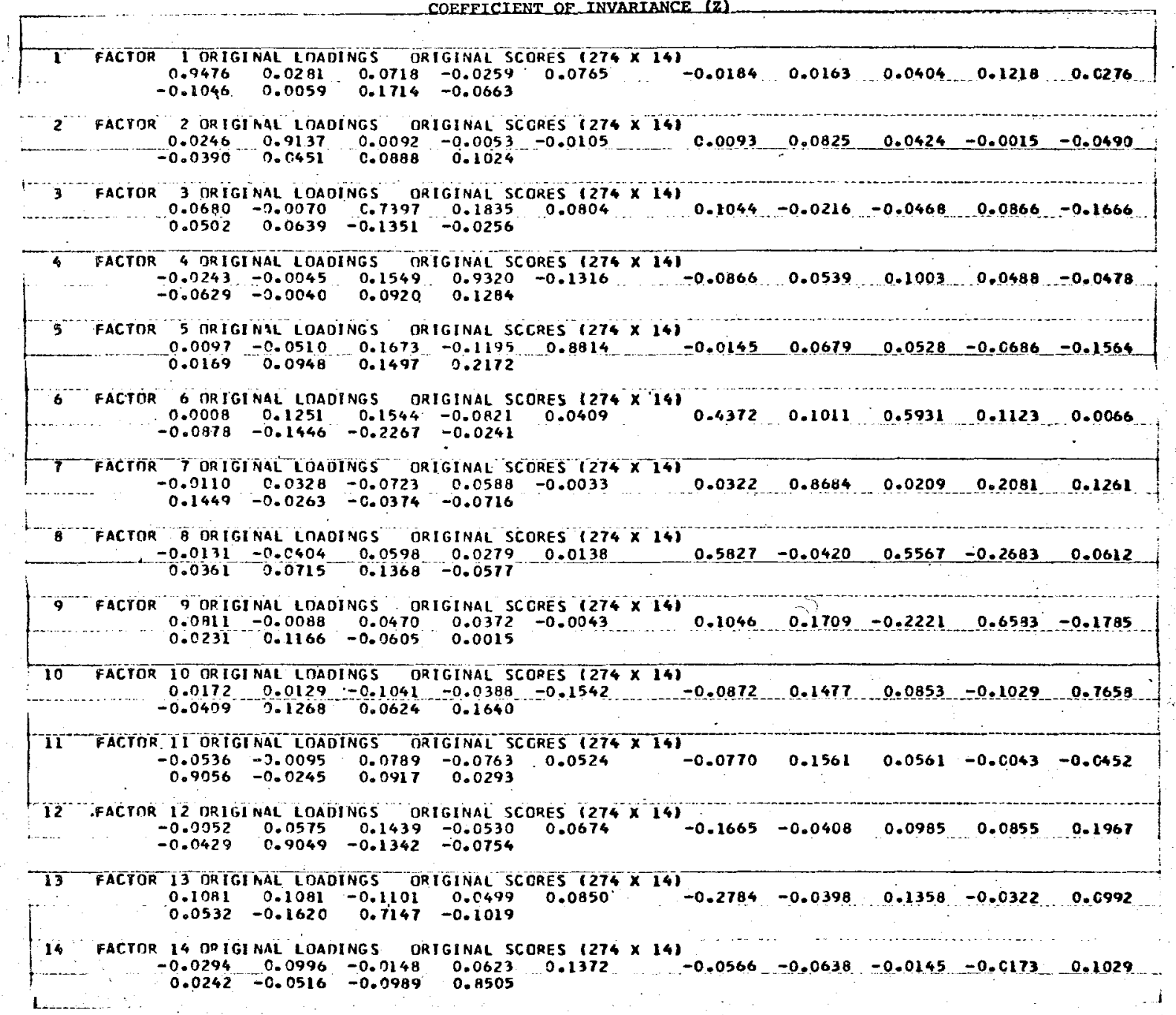


TABEF?

ORIGINAL : SCORES AND PERMUTED_SCORES_-_308_COUUMNS

COEFFICIENT OF. INVARIANCE (Z")

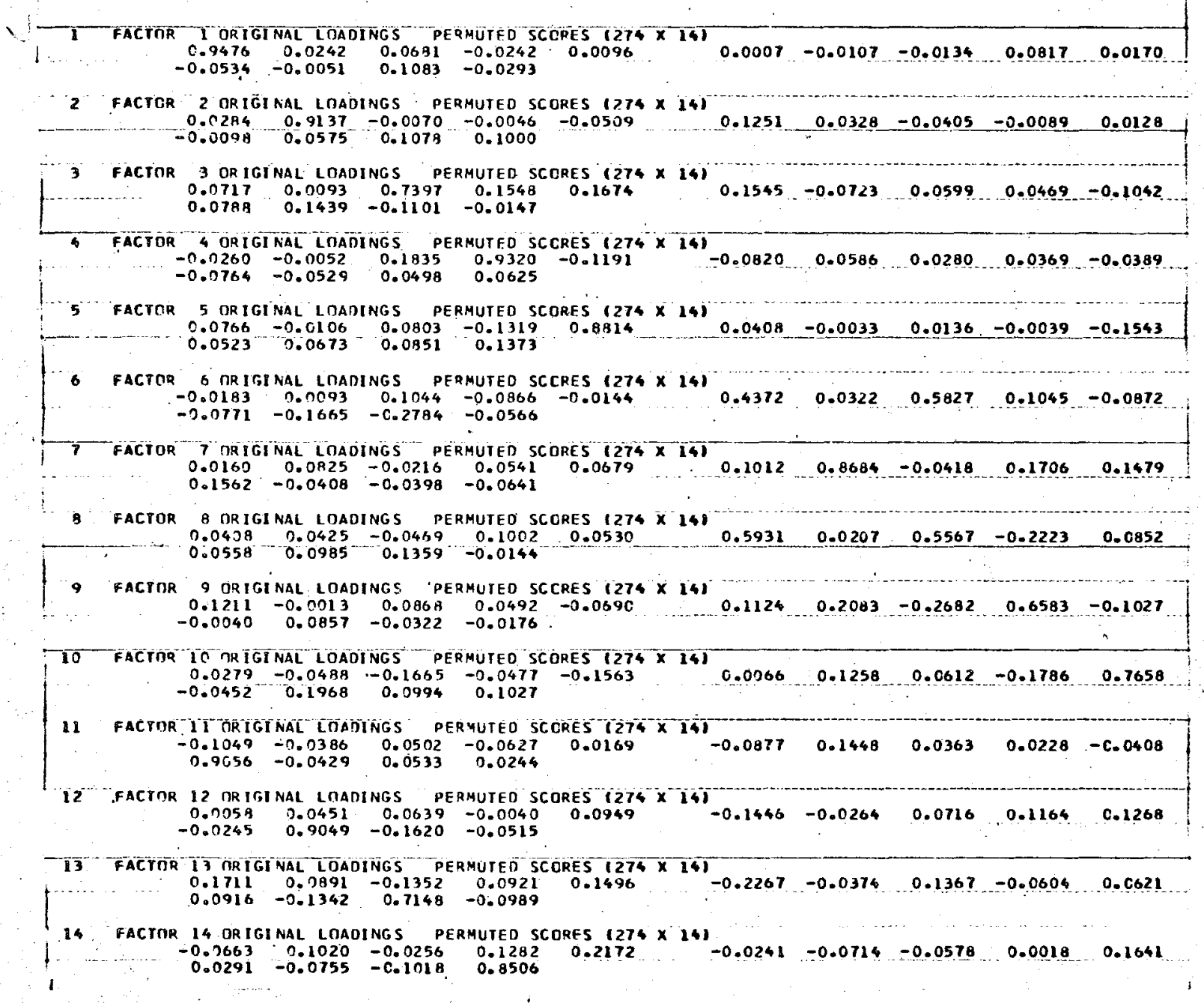


TABLE- 20

ORIGINAL SCORES AND. PERMUTED.SCORES-30R COLWANS

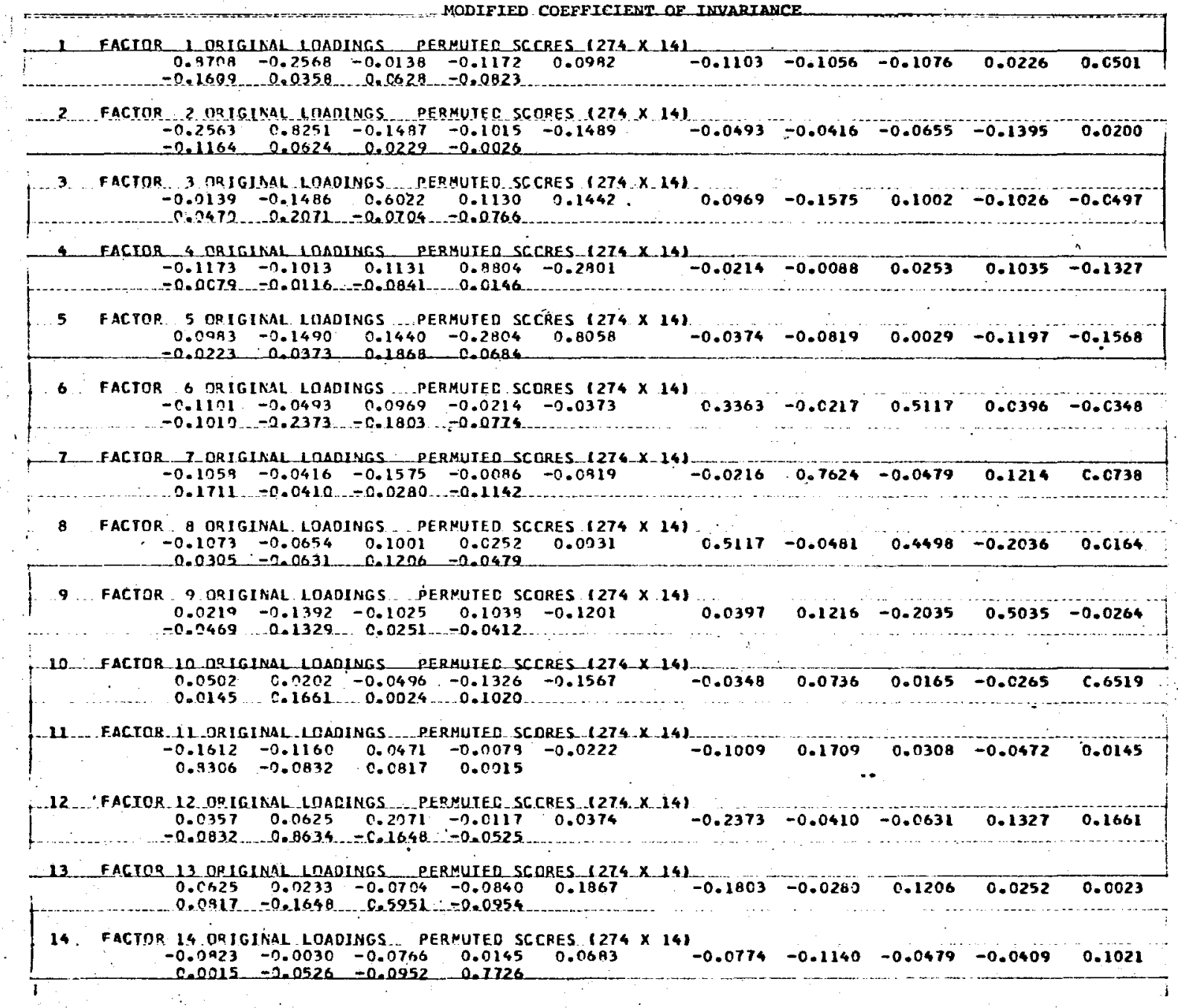


reflect the increasing disparity between the two sets of factors. That is, the relationship between the original factors and the factors based on $60 \%$ permuted $\mathrm{Z}$ should decrease from the previous results of the $30 \%$ permutation case.

Coefficient of congruence. This measure resulted in an identity matrix.

Coefficient of invariance. Tables 11 and 12 present the results for both solutions of the coefficient of invariance. In neither case are the results an identity matrix. A comparison of Table 11 and 12 with Tables 8 and 9 presented earlier for the $30 \%$ column permutation case, shows that the relationships between the factors are lower when more columns of $\mathrm{z}$ are permuted. As was true in the preceding case, one solution is the transpose of the other. Modified coefficient of invariance. It was not possible to compute the empirical results for the modified coefficient of invariance in this case. ${ }^{2}$ However, other evidence indicates that in this case results for the modified coefficient should be consistent with those expected for an adequate measure of invariance. This evidence consists of results for the case of identical scores and procedures, the case of $30 \%$ column permutation, the cases of $30 \%$ and $100 \%$ row and column permutation

2 These results were unavailable due to a complete changeover in operating systems at Western Data Processing Center. 
TARLE 21.

ORIGINAL SCORES AND PERMUTED. SCORES. = 608 COLURAS

COEFFICIENT OF INYARIANCE (z)

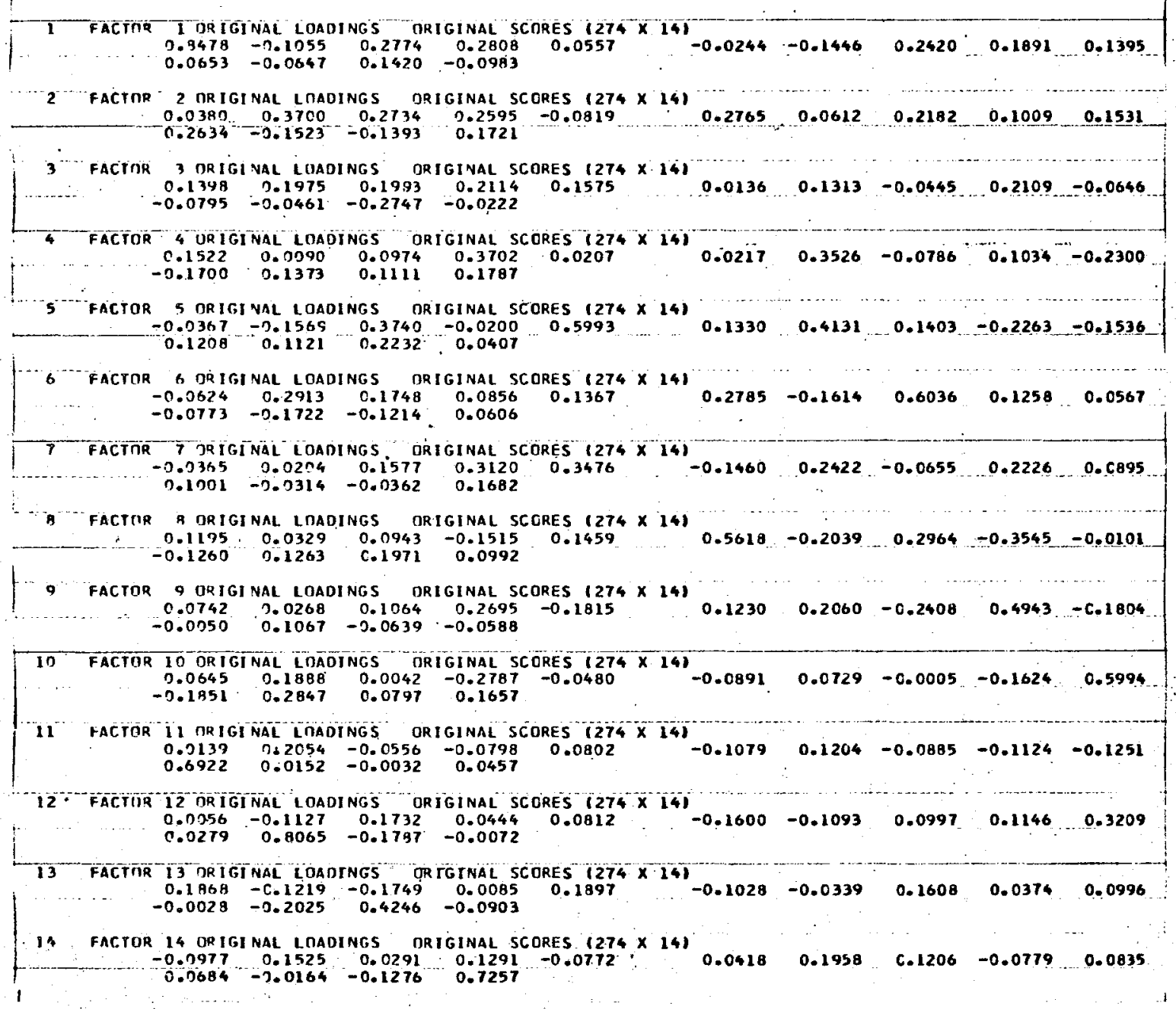


TABLE 12

ORIGINAS SCORES AND PERMUTED SCORES - 601 COLUMNS

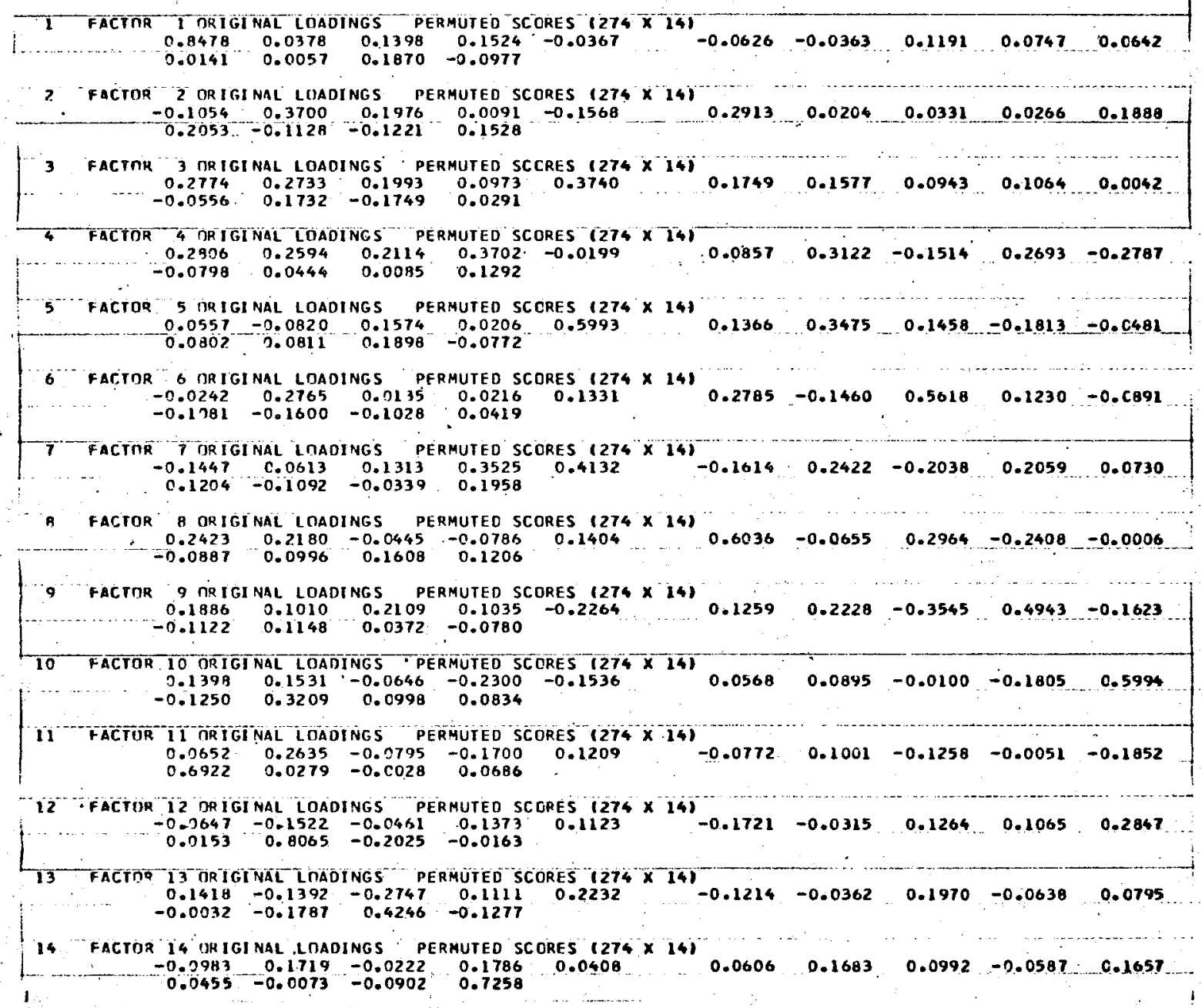
COEFFICIENT OF INVARIANCE (Z*) 
presented in a later section of this paper, and additional interpretation of equations presented in the summary of results for this section.

\section{0\% Column permutation}

The results expected for an adequate measure of invariance in this case are similar to the results of the $30 \%$ and $60 \%$ column permutation cases, i.e., the relationships between the two sets of factors should not be an identity matrix. In addition, since more columns are permuted in this case, the other criterion for an adequate measure of invariance is that the relationships between the factors will be even lower than they were for $30 \%$ and $60 \%$ column permutation.

Coefficient of congruence. The result for this measure is an identity matrix.

Coefficient of invariance. Tables 13 and 14 show that the coefficient of invariance does not result in an identity matrix. Tables 13 and 14 , when compared with.. Tables 11 and 12 of the $60 \%$ column permutation case and Tables 8 and 9 of the $30 \%$ column permutation case, show that the similarity between the two sets of factors is lowest when $90 \%$ of the columns of $z$ are permuted. Again, Tables 13 and 14 indicate that in this case $R_{I V_{Z}^{\prime}}=R_{I V_{Z^{*}}}$; i.e., one matrix is the transpose of the other. These tables show that only Factors $I, X, X I I$, and XIV remain most highly related to Factors $I^{\prime}, X^{\prime}, X^{\prime} I^{\prime}$, and XIV', 
TABLEE 13

QRIGINAL SCORES AND PERMUTED SCORES - 908 COLUMNS

COEFPICIENT OF INVARIANCE (z)

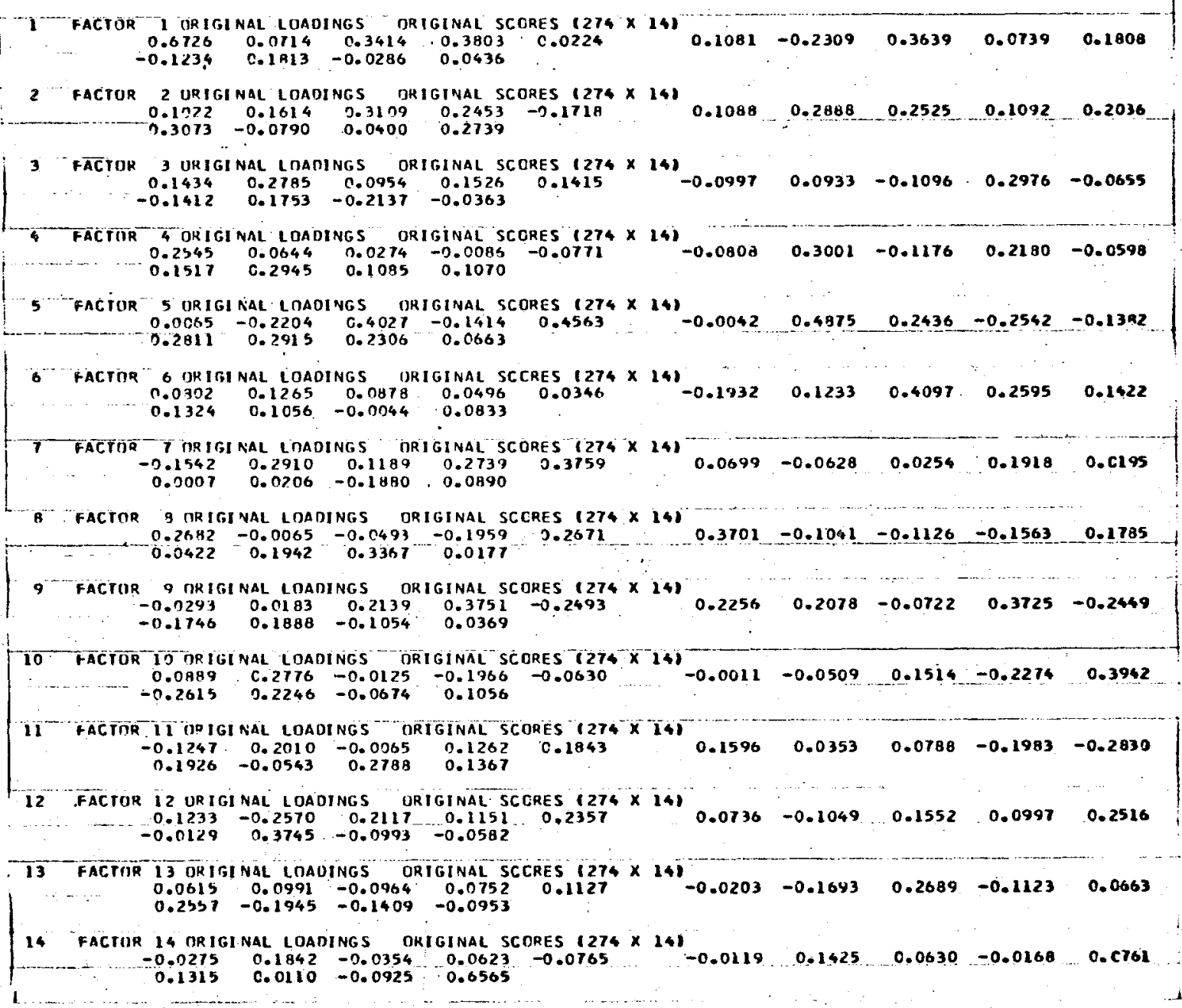


TABLE 14

ORIGINAL SCORES AND PERMUTED SCORES - 901 COLUMNS 
respectively; the remaining factors show highest relationships in the off diagonals.

Modified coefficient of invariance. The same circumstances hold for the modified coefficient of invariance in this case, as were presented in the $60 \%$ column permutation case. That is, the empirical results are not available but may be inferred on the basis of results already presented as well as the relationships described in the summary of results for this section.

Summary of Results for Column Permutations

For $30 \%$ column permutation the coefficient of congruence was an identity matrix. Increasing the number of permuted columns to $60 \%$ and $90 \%$ did not change the results:

In each of the three cases of permuted columns the coefficient of invariance did not result in an identity matrix. The coefficient of invariance did show that as additional columns of $\mathrm{z}$ were permuted the relationships. between the two sets of factors decreased. Consequently, the results presented for the coefficient of invariance conform to the results expected for an adequate measure of invariance in the case of permuted columns. An additional result of the coefficient of invariance was that for each of the three cases of column permutation the matrix of coefficients of invariance based on $z$ was equivalent to the transpose of the matrix of coefficients based on $z^{*}$. The 
following equations show that $R_{I V_{Z}^{\prime}}=R_{I V_{Z^{*}}}$. By definition $L^{*}=P L$ where $L$ is the original set of factor loadings. $\mathrm{P}$ is a permutation matrix; hence, $\mathrm{P}=\mathrm{P}^{\prime}=$ $\mathrm{P}^{-1}$. Substituting for $\mathrm{L}^{*}$ in equation 2 shows that

$$
\text { [7] } \begin{aligned}
\mathrm{R}_{I V_{Z}} & =L^{\prime}(\mathrm{PL})^{-1 !} \\
& =L^{\prime} \quad \mathrm{PL}^{-{ }^{\prime}}
\end{aligned}
$$

By substitution in equation 3

$$
\text { [8] } \quad R_{I V_{Z^{*}}}=\mathrm{L}^{-1} \mathrm{PL} \text {. }
$$

Transposing $R_{I V_{Z}}$ yields

$$
{ }^{\mathrm{R}} \mathrm{IV}_{\mathrm{Z}}^{\prime}=\mathrm{L}^{\prime} \mathrm{PL}^{-\mathrm{I}^{\prime},}
$$

which is equal to $R_{I V_{Z^{*}}}$. Thus, $R_{I V_{Z}^{\prime}}={ }_{I V_{Z^{*}}}$.

Results for the modified coefficient of invariance in the case of $30 \%$ column permutation did not reveal an identity matrix. These résults, presented in Table 10, indicate that a modified coefficient is consistent with the criteria for an adequate measure of invariance in the case of column permutation. The equations for the modified coefficient are as follows:

$$
\begin{aligned}
R_{I V}^{\circ} & =\frac{I}{N} S^{0}, S^{* 0} \\
& =\frac{I}{N} I^{-1} \mathrm{Z}^{0}, \mathrm{Z}^{0} \mathrm{~L}^{*^{-I^{\prime}}} \\
& =I^{-1} I \mathrm{I}^{-1}{ }^{\prime}
\end{aligned}
$$




$$
=L^{-1} L^{*-1} \text {, }
$$

Since $\mathrm{I}^{*}=\mathrm{PL}$, by substitution,

$$
\text { R } \dot{I V}^{\circ}=\mathrm{L}^{-1} \mathrm{P} \quad \mathrm{I}^{-1}
$$

It is evident from the last equation that as the permutation matrix (P) becomes increasingly disparate from I, $\mathrm{L}^{-1}$ and $\mathrm{L}^{*-1}$ also become increasingly different. Consequently, their product departs further and further from an identity matrix. While, as noted earlier, it was not possible to obtain the empirical results for $60 \%$ and $90 \%$ column permutation, these considerations indicate that this measure agrees with the criteria for an adequate measure of invariance:

\section{Row and Column Permutation}

The criterion for an adequate measure of invariance in this case was not an identity matrix. In addition, it was expected that increasing the number of permuted rows and columns should reduce the magnitude of the coefficients presented for each measure of invariance. The advantage of this case lies in the fact that by permuting both rows and columns of $\mathrm{z}$ to form $\mathrm{Z}$ * more variation has been introduced between the two sets of $\mathrm{Z}$ scores.

30\% Row and Column Permutation

Coefficient of congruence. Table 15 presents the results of correlating the appropriate sets of factor 
TABLE 15

ORIGINAL SCORES AND RERMUTED SCORES - 308 ROWS.AND COLUMNS.

COEFFICIENT: OF CONGRUENCE

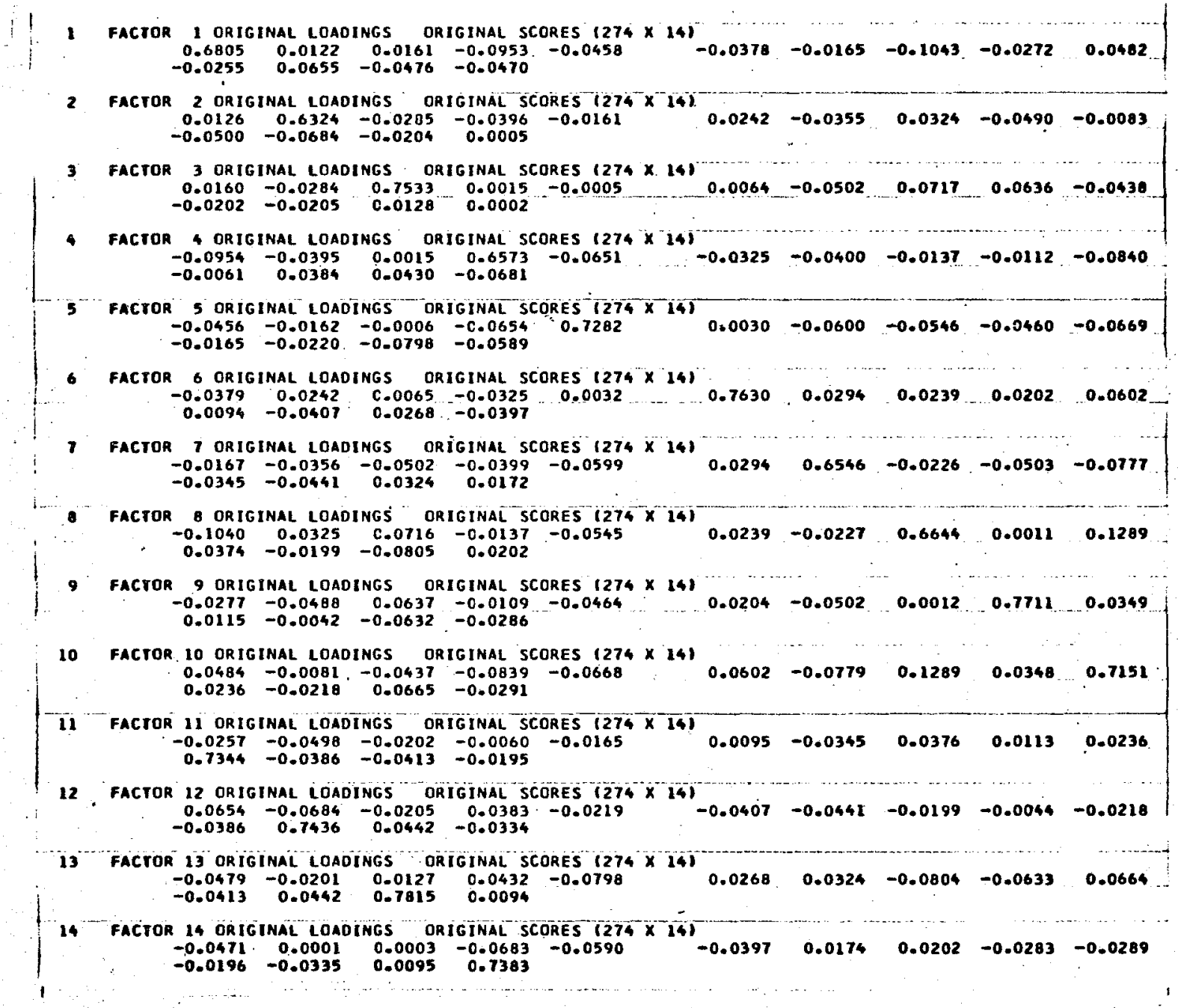


scores for the coefficient of congruence. These results do not indicate an identity matrix for this case.

Coefficient of invariance. Tables 16 and 17 present the two solutions for measuring the relationship between the appropriate set of factor scores. Neither table is an identity matrix. In addition, the matrix of coefficients of invariance based on $\mathrm{z}, \mathrm{Table} 16$, is the transpose of the matrix of coefficients based on $Z^{*}$, Table 17 .

Modified coefficient of invariance. Table 18 shows

the results of the modified coefficient of invariance when $30 \%$ of the rows and columns of $\mathrm{z}$ are permuted. Again, the results presented in Table 18 do not show an identity matrix.

60\% Row and Column Permutation

In this case, an additional criterion was established for an adequate measure of invariance; that is, it is expected that the degree of relationship between the two sets of factors should be lower than it was in the previous case of $30 \%$ row and colúmn permutation.

Coefficient of congruence. Table 19 presents the results for this case. This measure does not produce an identity matrix. A comparison of Table 19 with Table 15, the results of the coefficient of congruence for $30 \%$ row and column permutation, reveals that the coefficients are lower for $60 \%$ row and column permutation.

Coefficient of invariance. The two solutions 
TABLE 16

ORIGINAL SCORES AND PERMUTED SCORES - 308 ROWS. AND COLUMNS.

COEFFICIENT OF INVARIANCE (z)

1 FACTOR 1 ORIGIMAL LOADINGS DRIGINAL SCORES $(274 \times 14)$

| $\quad \begin{array}{rllllllllllll}0.9476 & 0.0281 & 0.0718 & -0.0259 & 0.0765 & -0.0186 & 0.0163 & 0.0404 & 0.1218 & 0.0276\end{array}$

2 TACTOR 2 ORIGINAL LOAOINGS ORIGINAL SCORES $(274 \times 14)$

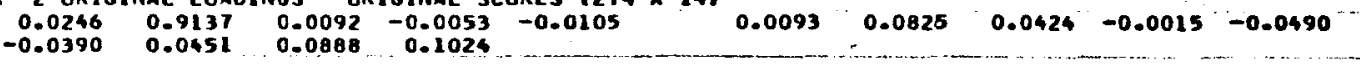

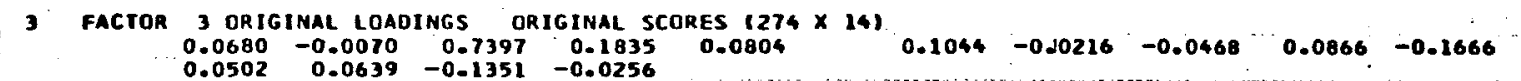

4 FACIOR 4 ORIGINAL LOADINGS ORIGINAL SCORES $(274 \times 14)$ $\begin{array}{llllllllllllllllll}-0.0243 & -0.0045 & 0.1549 & 0.9320 & -0.1316 & -0.0866 & 0.0539 & 0.1003 & 0.0488 & -0.0478\end{array}$ $-0.0629-0.0040 \quad 0.0920 \quad 0.1284$

5 Factior 5 original loaOings original SCORES $(274 \times 14)$

$\begin{array}{lllllllllllllll}0.0097 & -0.0510 & 0.1673 & -0.1195 & 0.8814 & -0.0145 & 0.0679 & 0.0528 & -0.0686 & -0.1564\end{array}$ $\begin{array}{lllllllllll}0.0097 & -0.0510 & 0.1673 & -0.1195 & 0.8814 & -0.0145 & 0.0679 & 0.0528 & -0.0686 & -0.1564 \\ 0.0169 & 0.0948 & 0.1497 & 0.2172 & & \end{array}$

6 factor 6 original loadings original scores $(274 \times 14)$ $\begin{array}{lllllllllll}0.0008 & 0.1251 & 0.1544 & -0.0821 & 0.0409 & 0.4372 & 0.1011 & 0.5931 & 0.1123 & 0.0066\end{array}$ $-0.0878-0.1446-0.2267-0.0241$

7 FACTOR, 7 ORIGINAL LOAOINGS ORIGINAL SCCRES $(274 \times 14)$ $\begin{array}{llllllllllllllll}-0.0110 & 0.0328 & -0.0723 & 0.0588 & -0.0033 & 0.0322 & 0.8686 & 0.0209 & 0.2081 & 0.1261\end{array}$ $0.1449-0.0263-0.0374,-0.0716$

- factor a original loadings original scores $(274 \times 14)$ $\begin{array}{llllllllll}-0.0131 & -0.0404 & 0.0598 & 0.0279 & 0.0138 & 0.5827 & -0.0420 & 0.5567 & -0.2683 & 0.0612\end{array}$ 0.03610 .0715

9 FACTOR 9 ORIGINAL LOAOINGS ORIGINAL SCORES $(274 \times 14)$ $\begin{array}{lllllllllll}0.0811 & -0.0088 & 0.0470 & 0.0372 & -0.0043 & 0.1046 & 0.1709 & -0.2221 & 0.6583 & -0.1785\end{array}$ $0.02310 .1166 \quad-0.0605 \quad 0.0015$

10 FACTOR 10 ORIGINAL LOADINGS ORIGINAL SCORES $(274 \times 141$ $\begin{array}{llllllllllllll}0.0172 & 0.0129 & -0.1041 & -0.0388 & -0.1542 & -0.0872 & 0.1477 & 0.0853 & -0.1029 & 0.7658\end{array}$ $-0.04090 .12680 .0624 .0 .1640$

11. FACTOR. 11 ORIGINAL LOADINGS ORIGINAL SCGRES $(274 \times 24)$ $\begin{array}{llllllllllllllll}-0.0536 & -0.0095 & 0.0789 & -0.0763 & 0.0524 & -0.0770 & 0.1561 & 0.0561 & -0.0043 & -0.0452\end{array}$ $\begin{array}{llll}0.9056 & -0.0245 & 0.0917 & 0.0293\end{array}$

22 FACTOR 12 ORIGINAL LOAOINGS ORIGINAL SCORES $(274 \times 14)$

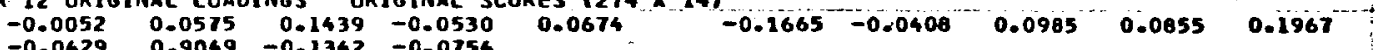

13 FACTOR 13 ORIGIMAL LOADINGS ORIGINAL SCORES $(274 \times 16)$

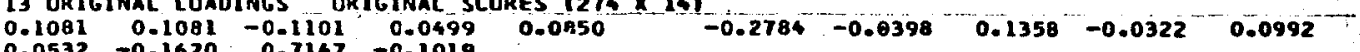

FACTOR 14 ORIGIMAL LOADINGS ORIGIMAL SCORES $(274 \times 14)$

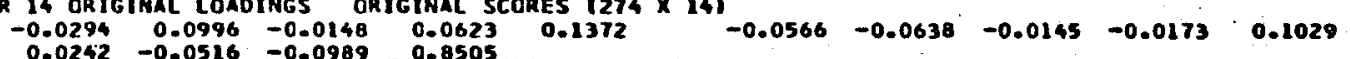


TABLE 27

ORIGINAT SCORES AND PERMUTED SCORES - 308 ROWS AND COLUMNS

COEFFICTENT OF INVARTANCE (2*)

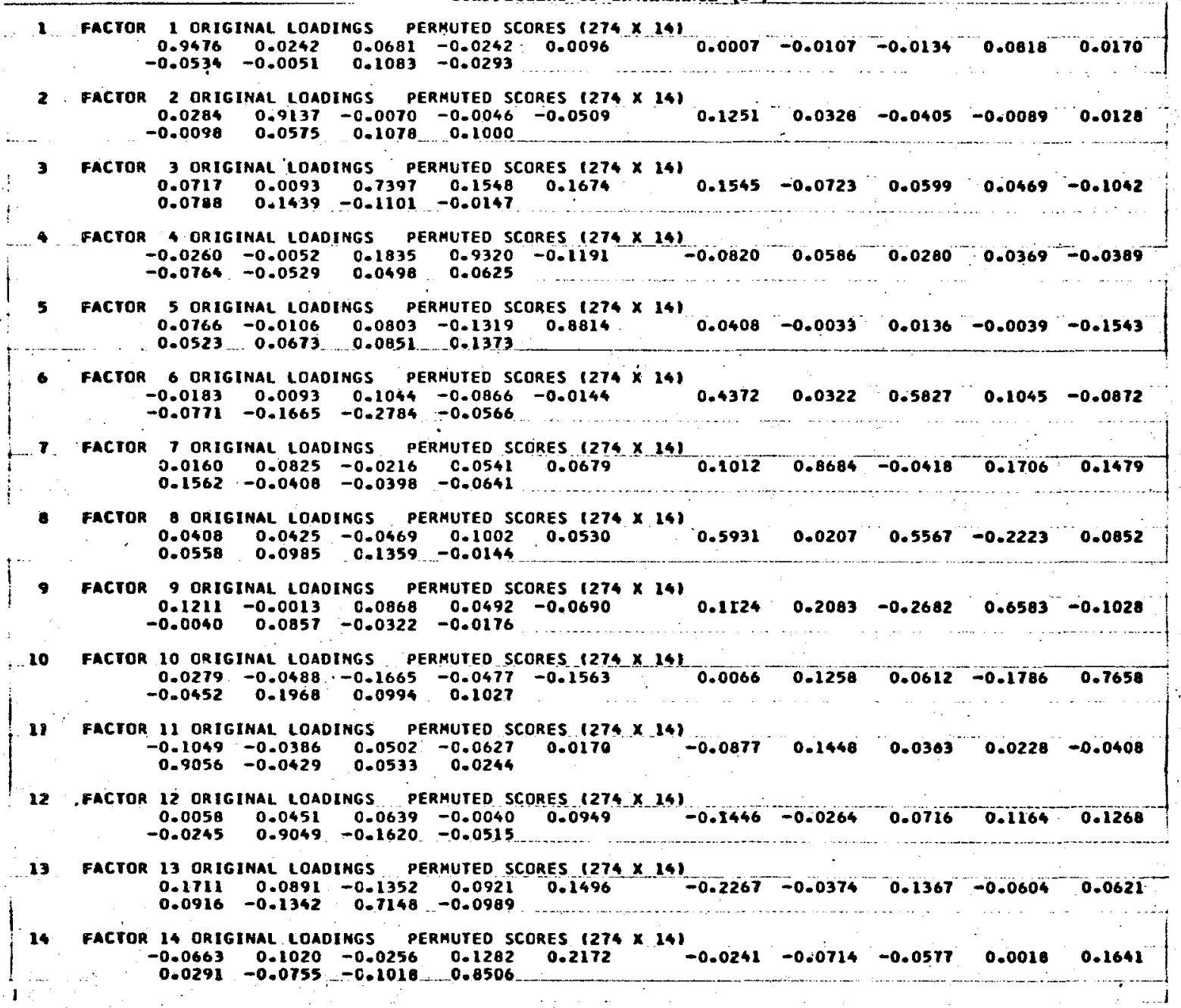


TABLE 18

ORIGINAL_SCORES AND PERMUTED SCORES .. 308 ROWS AND COLUMWS MODIFIED COEFFICIENT OF INVARIANCE

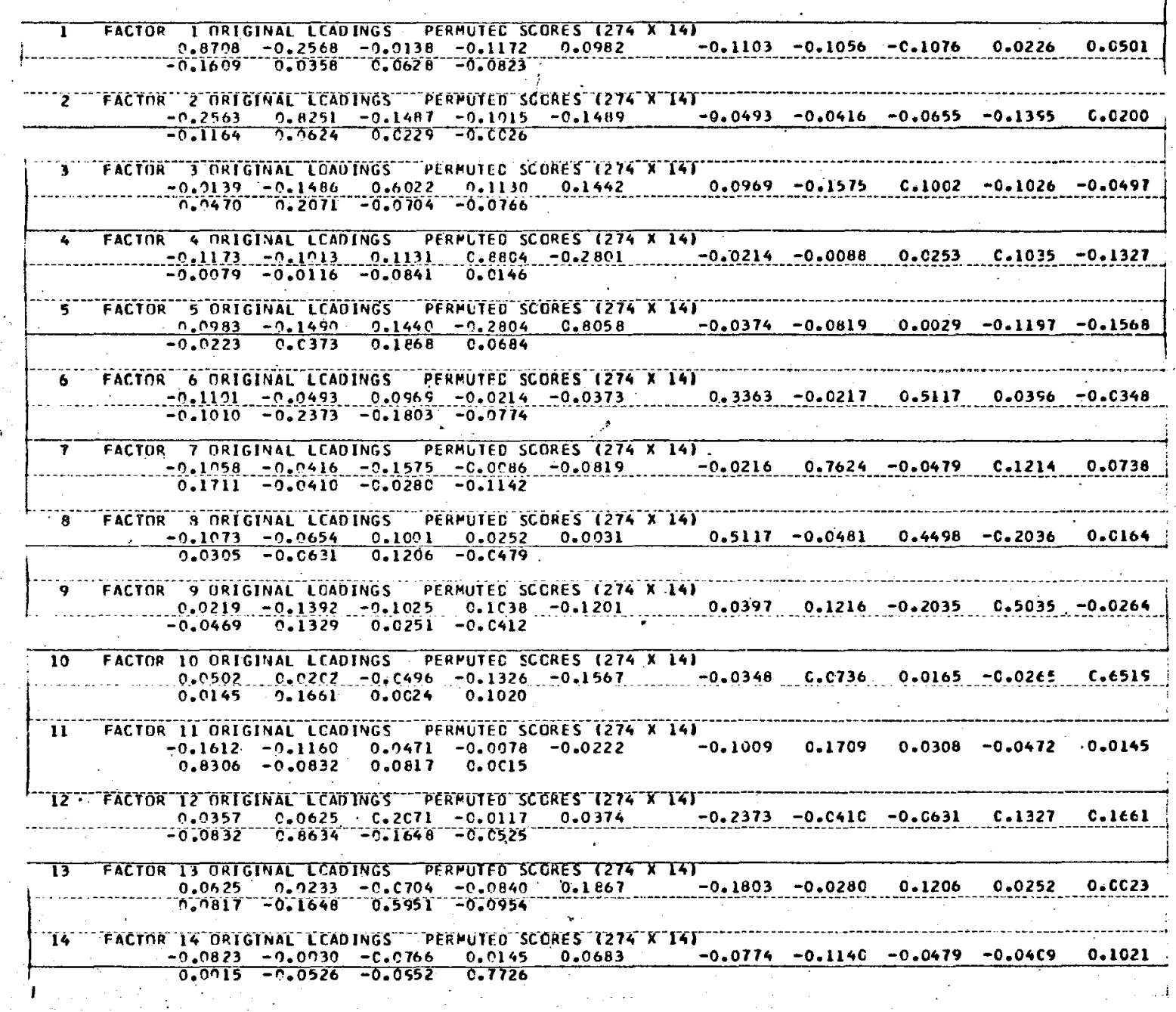


TABLE 19

ORIGINAL SCORES AND PERMUTER SCORES - 608 ROWS AND COLUMNS

COEFFICIENT OF CONGRUENCE

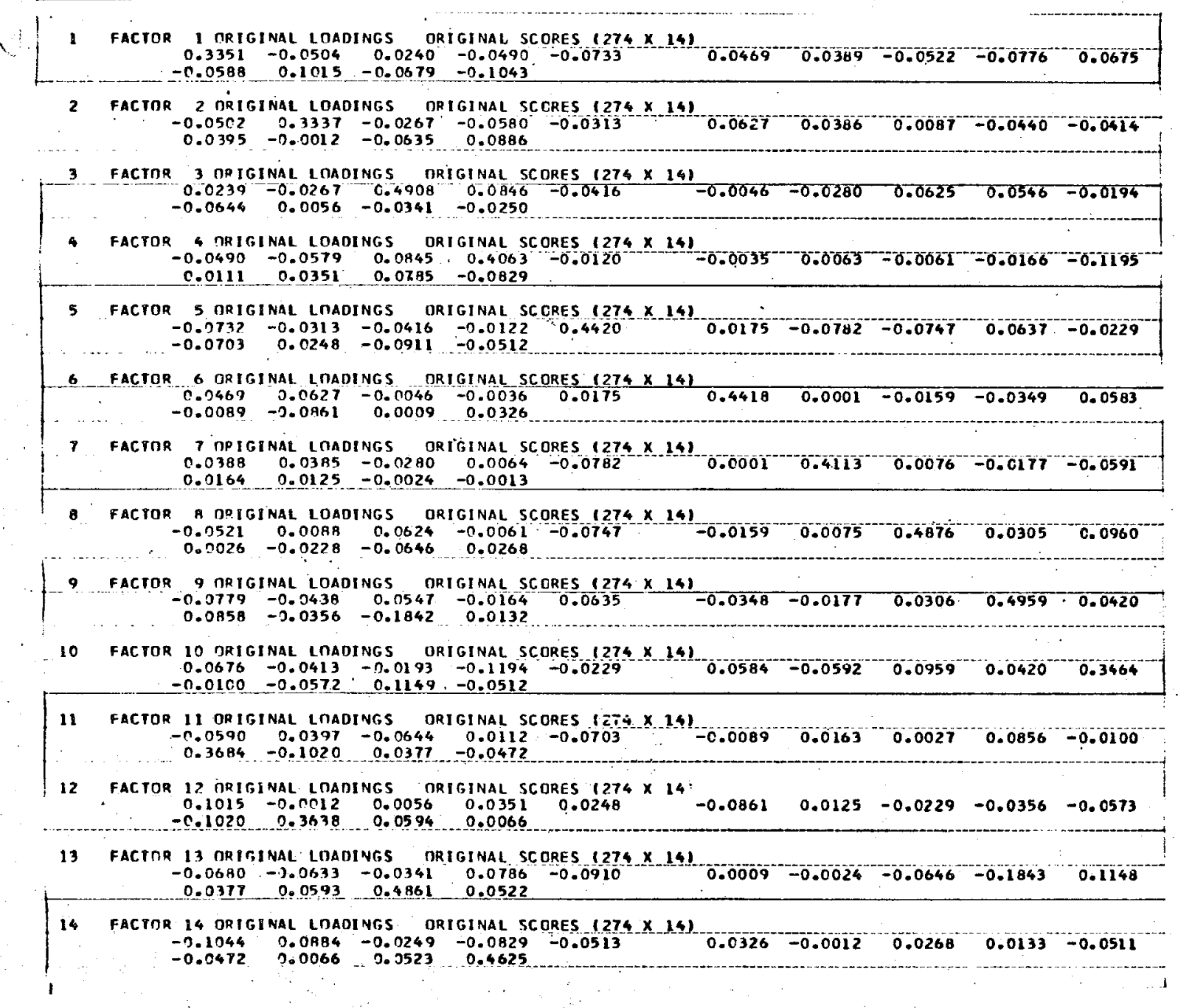


presented in Tables 20 and 21 also do not yield an identity matrix. A comparison of Tables 20 and 21 with the results of the $30 \%$ row and column permutation case, shown in Tables 16 and 17, indicates that permuting $60 \%$ of the rows and columns of $\mathrm{z}$ produces lower relationships between the factors than permuting $30 \%$ of the rows and columns of $z$. Again, one of the solutions for the coefficient of invariance is the transpose of the other; (cf. Tables 20 and 21 ).

Modified coefficient of invariance. Similar problems were encountered in this case as were true for $60 \%$ and $90 \%$ column permutation. That is, the empirical results were unavailable. Further implications of the modified coefficient of invariance.for the row and column permutation case are presented in the summary of results for this section.

\section{0\% Row and Column Permutation}

In this case, all the rows and columns of $\mathrm{z}$ are permuted to form $z^{*}$. The results predicted for an adequate measure of invariance were that the correlations between the appropriate factor scores for each coefficient should be due to chance alone. In other words, the relationships between the two sets of factor loadings have reduced to that expected by chance; therefore, an adequate measure of invariance is expected to reveal lower coefficients in this case than those presented for $30 \%$ and $60 \%$ row and column 
TABLE 20

ORIGINAL SCORES AND PERMUTED SCORES - 60 . ROWS AND. COLUMNS

COEFFICIENT. OF INUARIANCE ( $(x)$

I FACTIR 1 ORIGINAL LOADINGS ORIGINAL SCOQRES $(274 \times 14)$

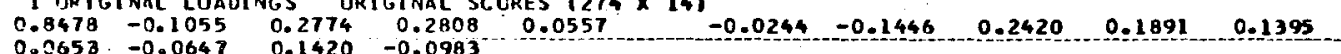

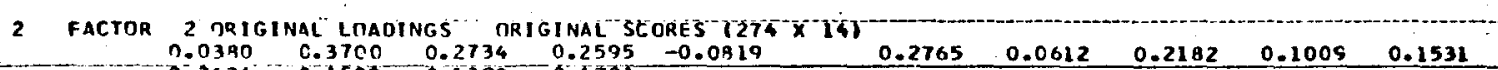
$0.2634-0.1523-0.1393-0.1721$

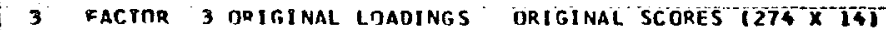

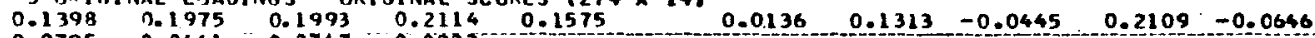
$-0.0795-0.0461-0.2747-0.0222$

4 FACTOA 4 OAP IGINAL LOADINGS ORIGINAL SCORES $1274 \times 161$

$\begin{array}{rrrrrrrr}0.1522 & 0.0090 & 0.0974 & 0.3702 & 0.0207 & 0.0217 & 0.3526 & -0.0786 \\ -0.1700 & 0.1373 & 0.1111 & 0.1787 & 0.1034 & -0.2300\end{array}$

5 FACTOR 5 ORIGINAL LOADINGS ORIGINAL SCORES $1274 \times 145$

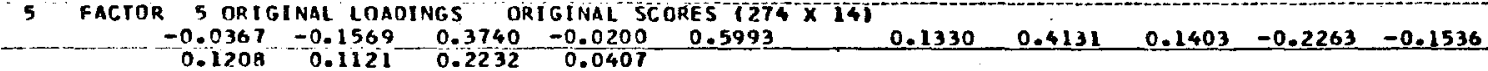

6 FACTOR 6 ORIGINAL LDADINGS ORIGINAL SCORES (274 $\mathrm{X}$ (4)

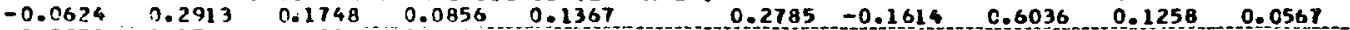
$-0.0773-0.1722-0.12140 .0606^{-1}$

7 FACTOR 7 ORIGTNAL LOADINGS ORIGINAL SCORES $1274 \times 14)$

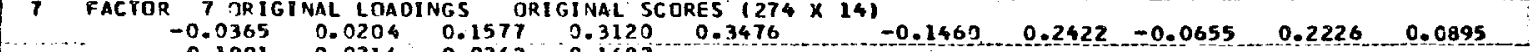

8 FACTOR 8 NRIGINAL LDADINGS ORIGINAT SCOEES $1274 \times 140$

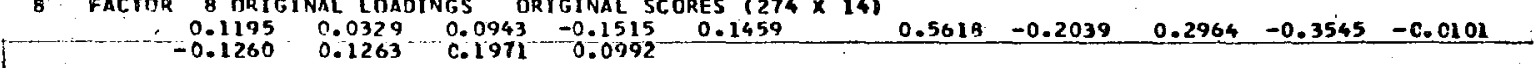

9 factirR 9 DRIGINAL LQADINGS ORIGINAL SCORES $1274 \times 141$ $0.0742 \quad 0.0268 \quad 0.1064 \quad 0.2695-0.1815 \quad 00.1230 \quad 0.2060-0.2408 \quad 0.4943-0.1804$ $-0.0050 \quad 0.1067-0.0639-0.0588$

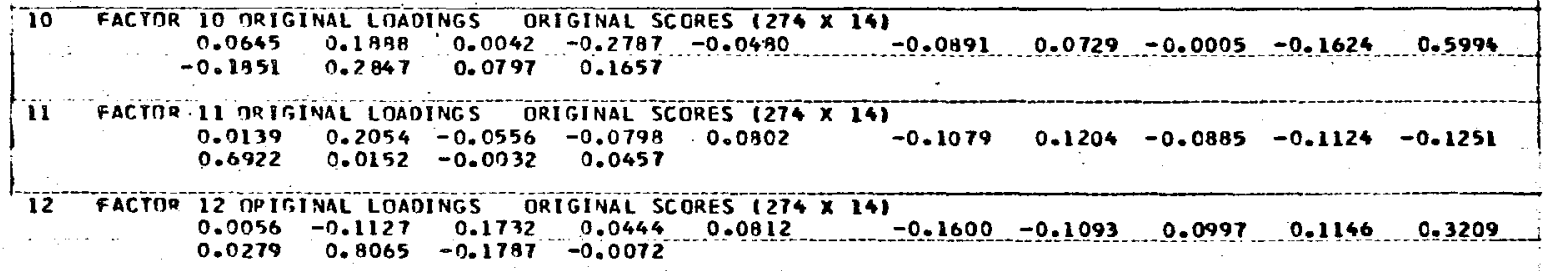

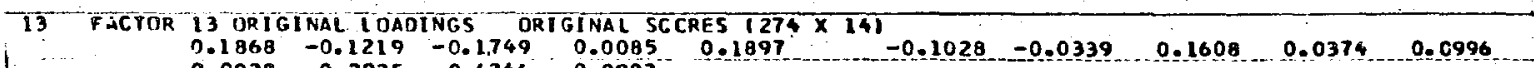
$\begin{array}{llll}-0.0028 & -0.2025 & 0.4246 & -0.0903\end{array}$

14 FACYTR 14 DRIGINAL LOADINGS DRIGINAL SCORES $1274 \times 145$

$\begin{array}{llllllllll}14 & 0.048 & 0.1206 & -0.0779 & 0.0835\end{array}$

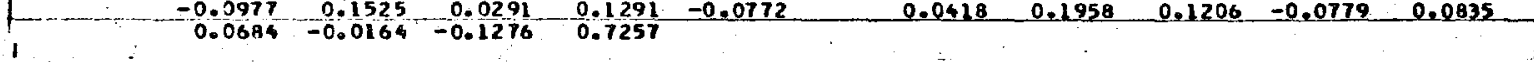


TABLE 21

ORIGINAL: SCORES AND PERMUTED. SCORES . -.. 60: ROWS AND COLURNS

COEFFICIENT OF INVARIANCE (Z*)

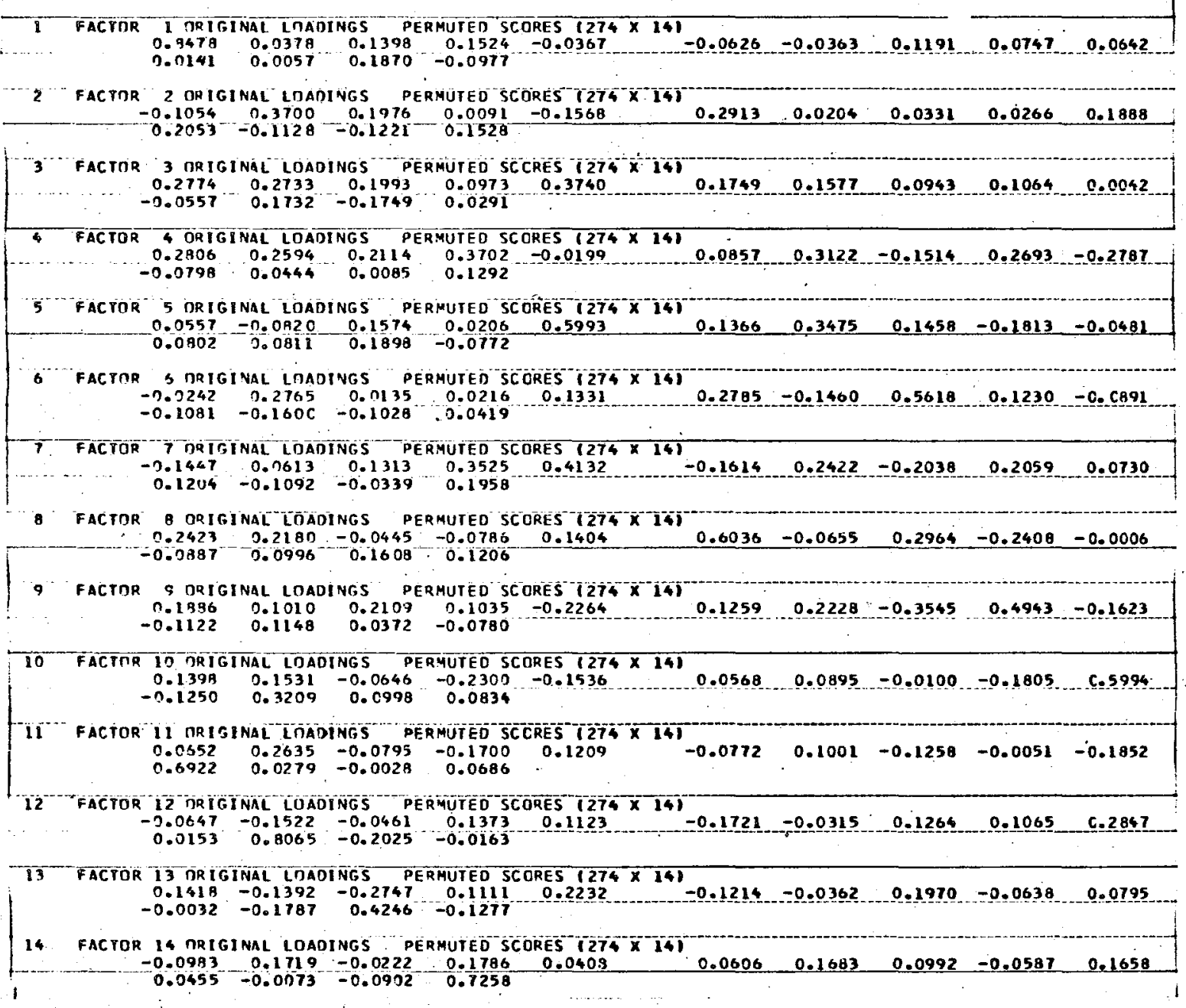


permutation.

Coefficient of congruence. The results for this measure are presented in Table 22. Table 22 indicates practically no relationship between the two sets of factors. The range of coefficients of congruence extend from -.0004 to .1329 .

Coefficient of invariance. Tables 23 and 24 show the two solutions for permuting $100 \%$ of the rows and columns of $\mathrm{z}$ to form $\mathrm{Z}^{*}$. These results indicate that the coefficient of invariance does not reflect consistent relationships between the two sets of factors. In addition, Tables 23 and 24 do show relationships which are lower than those presented for $30 \%$ and $60 \%$ row and column permutation. (Cf. Tables 16 and 17, and Tables 20 and 21). Again, one solution is the transpose of the other. The range of coefficients presented in Tables 23 and 24 extends from -.0068 to .5916 .

Modified coefficient of invariance. Table 25 presents the results for this measure. These findings indicate that the relationship between the factors is lower in this case than it was for $30 \%$ row and column permutation. The modified coefficients of invariance range from -.0027 to .4569 .

Summary of Results for All Five Criteria

None of the empirical findings for the three measures of invariance produced an identity matrix for any 


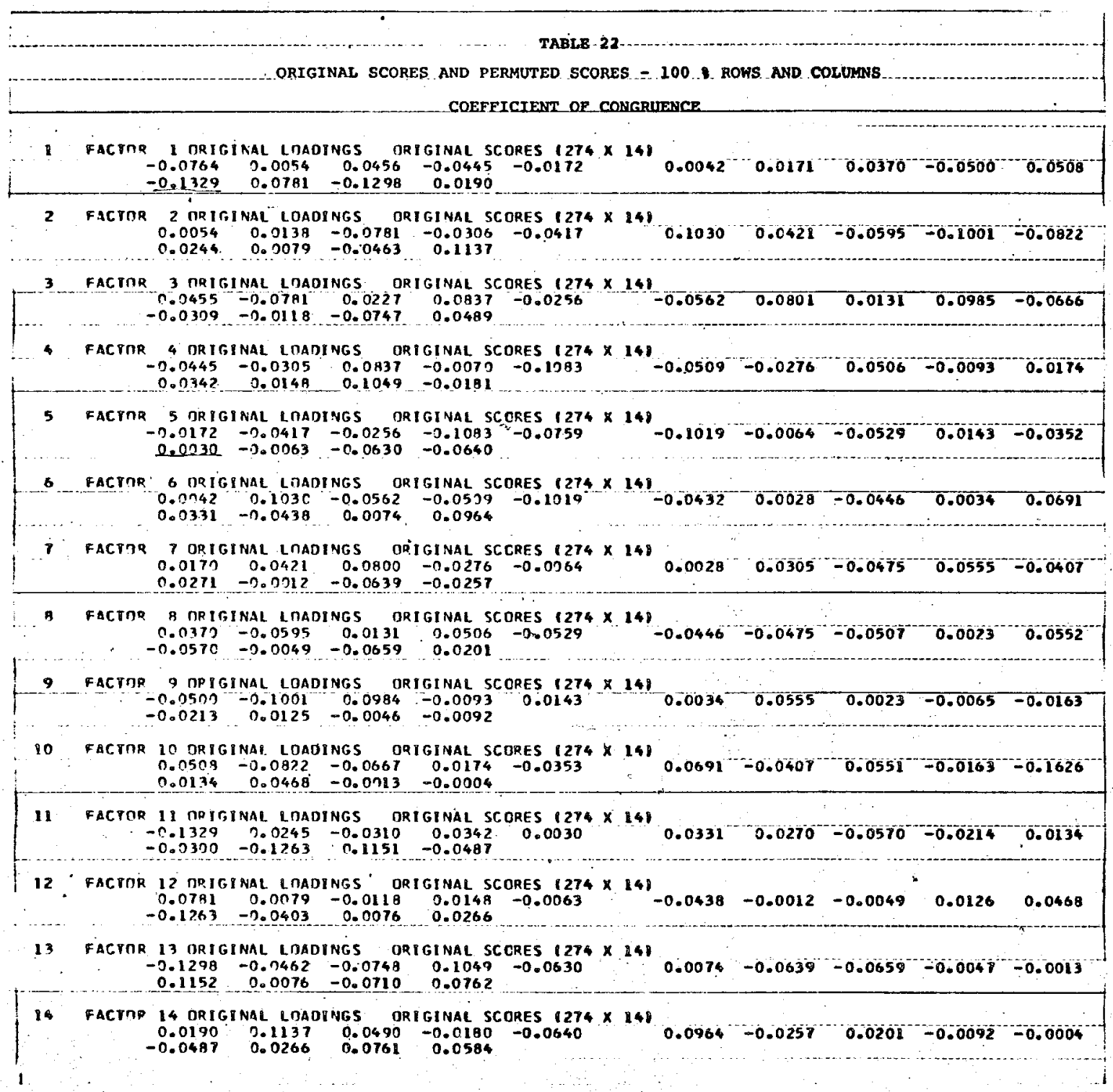


TABLE 23

ORIGINAL SCORES AND PERMUTED SCORES - 1008 ROWS AND COLUNAS COFFFICIENT OF INVARIANCE (z)

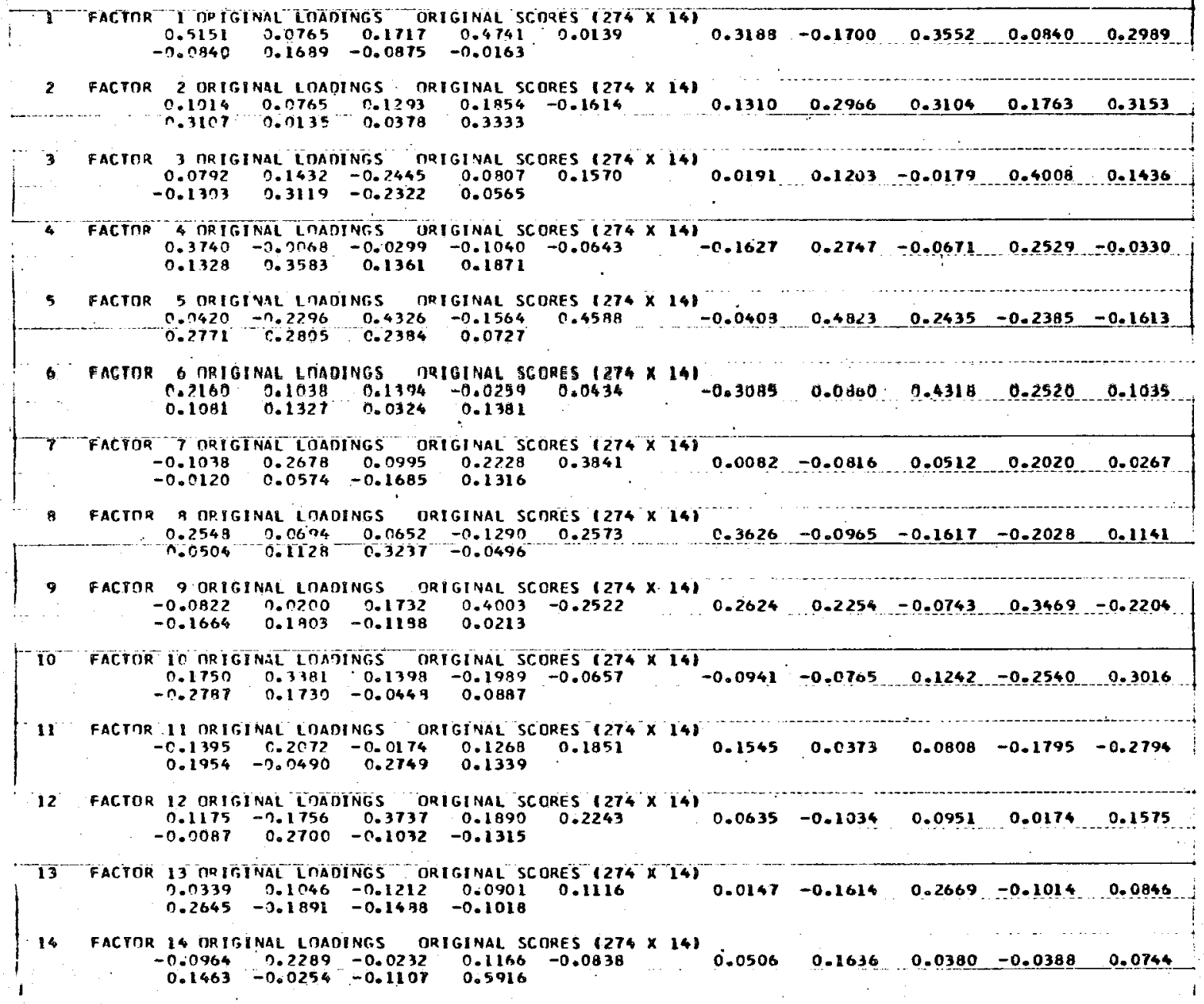


TABLE 24

ORIGINAL SCORES AND PERMUTED SCORES - 1008 ROWS AND COLUMNS COEFFICIENT OF INVARIANCE (Z*)

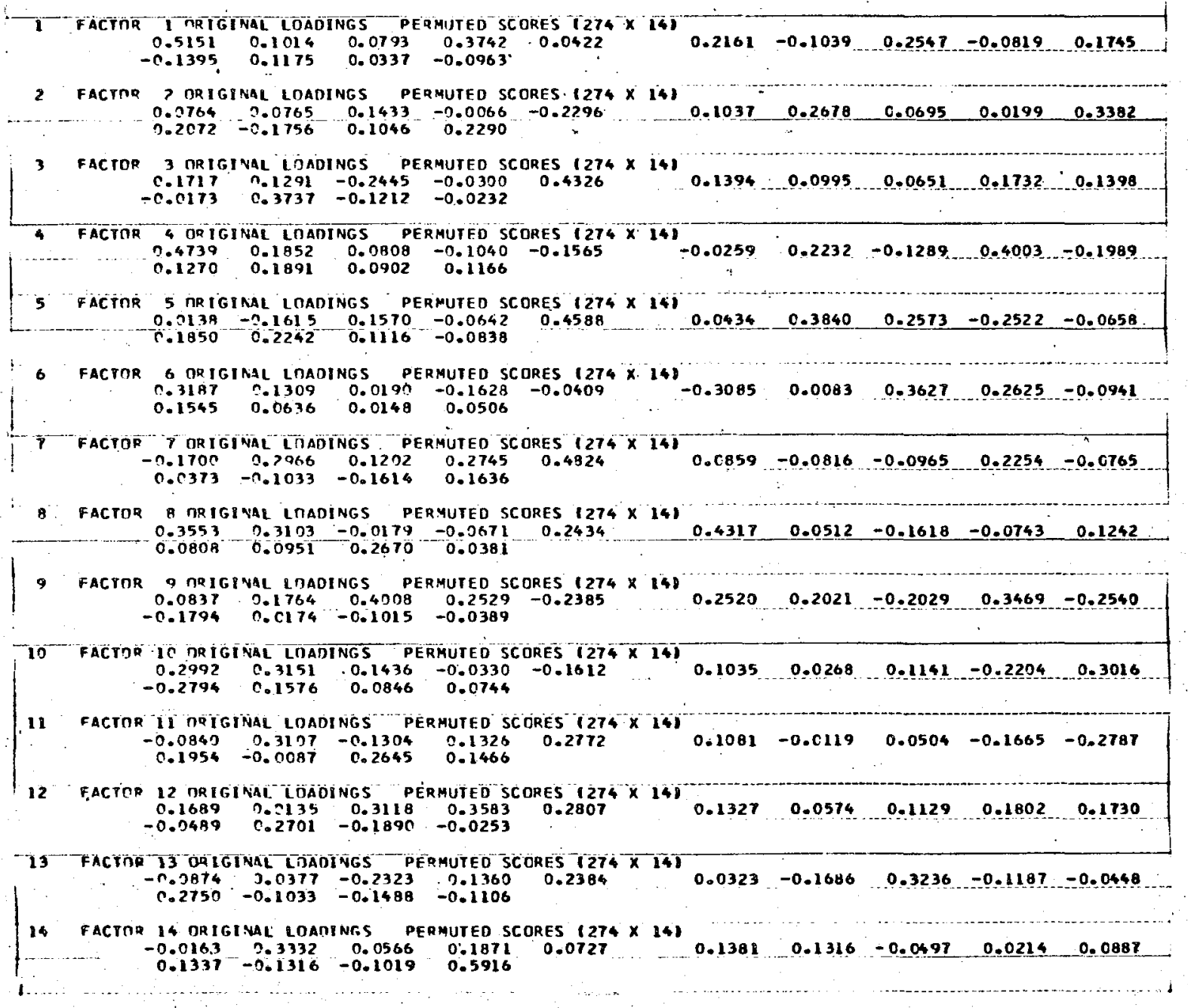


TABLE 25

_ORIGINAL_SCORES_AND_RERMUTED_SCORES_-_100Q_ROWS_AND_COLUMNS MODIEIED COEFEICIENT OE INVARIANCE

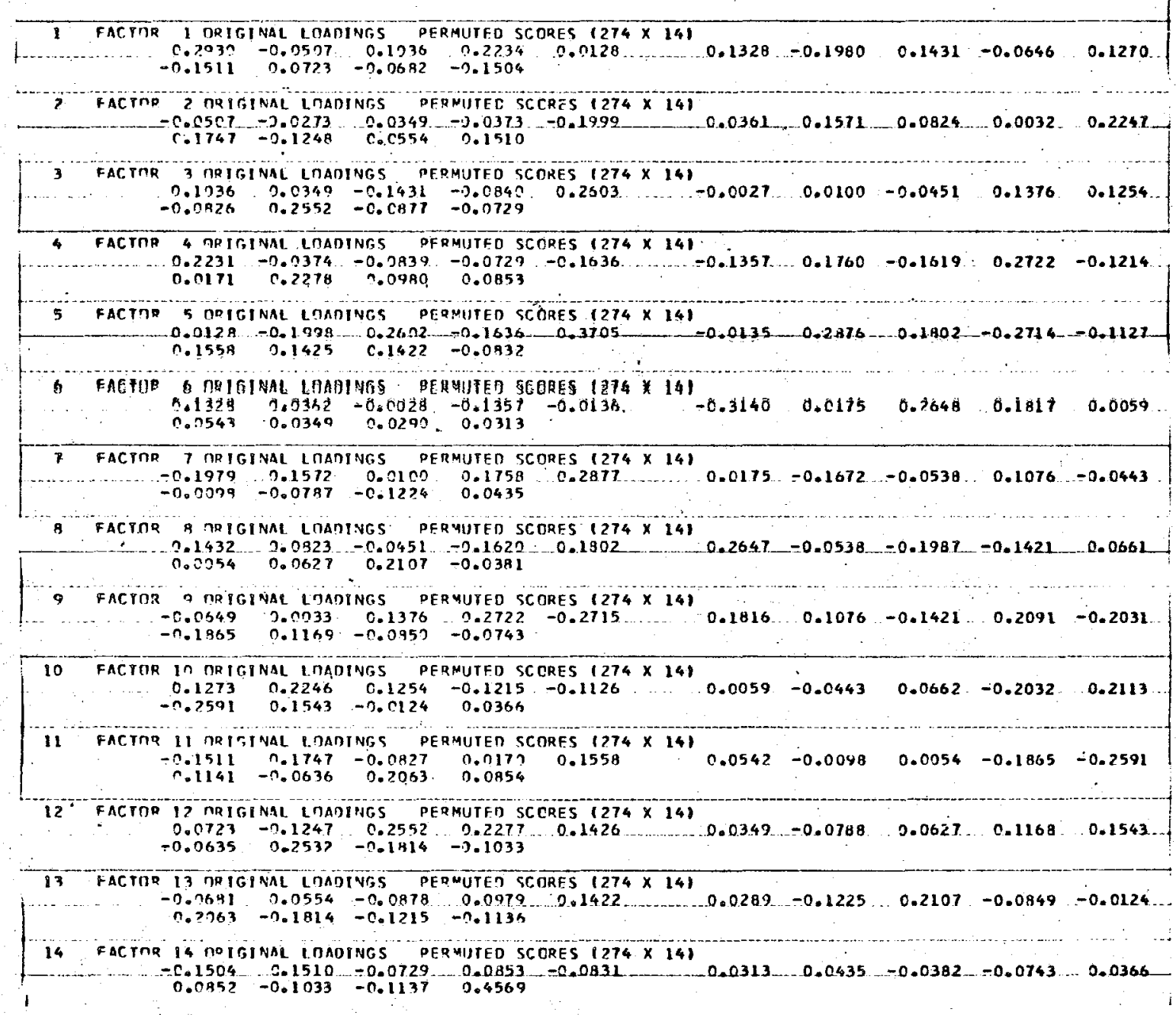


of the cases of row and column permutation. In this respect, all three measures agree with the criteria for an adequate measure of invariance. As more rows and columns of $\mathrm{z}$ were permuted, the relationships between the two sets of factors was shown to decrease by each of the measures. The expected result of permuting $100 \%$ of the rows and columns of $\mathrm{Z}$ to form $\mathrm{Z}^{*}$ was that an adequate measure of invariance, should reflect only chance relationships between the factors. The coefficient of congruence produced the lowest relationships. The coefficients of invariance were higher than either of the other two measures. Results for the modified coefficient of invariance were lower than the coefficient of invariance but slightly higher than the coefficients of congruence.

\section{Typical study}

For each of the cases mentioned previously the original matrix of factor loadings was derived from data collected on the initial day of a two week Head start

training program. The second set of factor loadings was computed from data collected on the last day of the training program. This second set of data consisted of responses of the same subjects to the same attitude survey. Thus, results are presented for a typical application of the fixed-variable, fixed-subject design.

Results Based on Principal Components Loadings Coefficient of Congruence. Table 26 presents the 


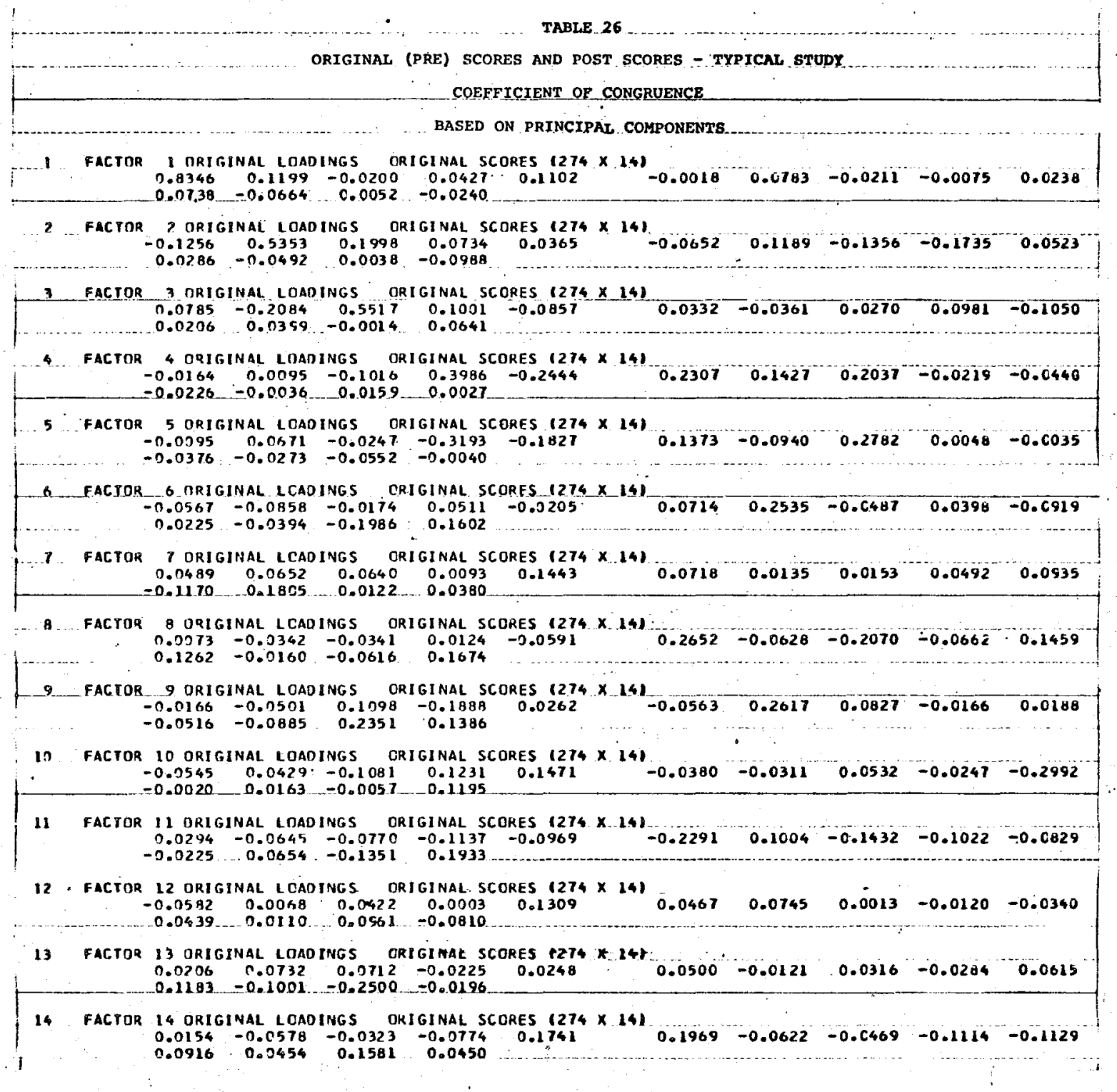


results of correlating the two appropriate sets of factor scores based on principal components loadings. These results indicate that factors I and I', II and II', and III and III' are highly related to each other. The coefficients presented in Table 26. range from .0003 to .8346.

Coefficient of Invariance. Tables 27 and 28 show the two solutions for this measure. Inspection of both sets of coefficients shows that factors I and I', II and II', III and III', IV and IV' and IV and $V^{\prime}$ and $V^{\prime}$ and $I V^{\prime}$ are highly related to each other. Inspection of both tables also indicates that the coefficients range from .0004 to .9931 . In the typical study one solution for the coefficient of invariance is not the transpose of the other. A comparison of Tables 27 and 28 reveals that although the numerical values are similar, the numerical values are not the same.

Results Based on Varimax Loadings

Modified coefficient of invariance. Table 29 presents the results of this measure for the typical case when the factor scores are based on varimax rotation of the principal components loadings. Factors I and I', IV and $V^{\prime}, V$ and $X I I I^{\prime}, V I$ and $I X^{\prime}, V I I$ and III', $X I$ and $X I^{\prime}$, and XII and $X^{\prime}$ are highly related to each other. The range of modified coefficients presented in Table 29 extends from .0004 to .7161.

Coefficient of Congruence. Table 30 shows the results 
TABLE . 27

ORIGINAL (PRE) SCORES AND POST SCORES - TYPICNL : STUDY COEFFICIENT OE INVARTANCE (Z)

BASFD ON PRINCIPAL COMPONENTS

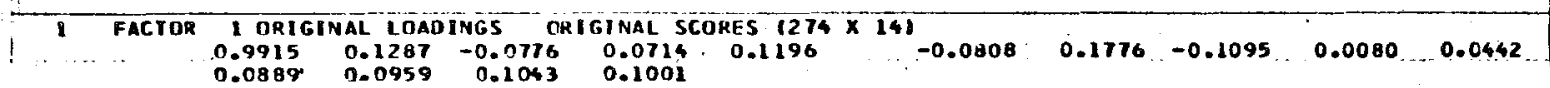

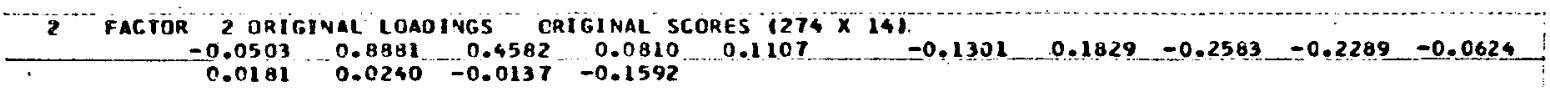

FACTOR TRIGINAL LOADINGS ORIGINAL SCCRES $(274 \times 141$

$\begin{array}{llllllllllll}0.0839 & -0.3640 & 0.8515 & 0.1088 & -0.0792 & -0.0360 & -0.1387 & 0.0952 & 0.2252 & -0.1270\end{array}$

4 FACTOR GORIGIVAL LOAOIVGS CRIGINAL SCORES $1274 \times 141$.

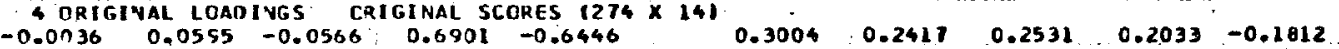
$\begin{array}{rrrr}-0.0036 & 0.0555 & -0.0566 & 0.6901 \\ 0.0642 & 0.1811 & 0.2373 & 0.0926\end{array}$

5 FACTDR 5 MRIGINAL LOAOINGS CRIGINAL SCCRES $(274 \times 14)^{2} 0.0869-0.1493 \quad 0.4422 \quad-0.0343-0.2619$ $\begin{array}{rrrr}0.0481 & 0.0499 & -0.0055 & -0.5645 \\ 0.0398 & -0.0139 & -0.1590 & 0.0247\end{array}$

6 Factor original lcadings orlainal scares $1274 \times 141$

$\begin{array}{lllllllllll}0.0304 & -0.1310 & 0.0239 & -0.0072 & 0.0379 & 0.1554 & 0.4917 & -0.2236 & -0.1716 & -0.4074\end{array}$ $-0.1733-0.2557-0.2271-0.00$

7 FACTOR 7 TRIGIMAL LOADINGS GRIGINAL SCCRES $1273 \times 141$

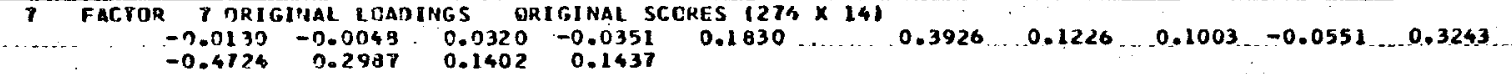

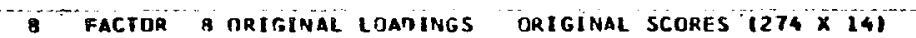

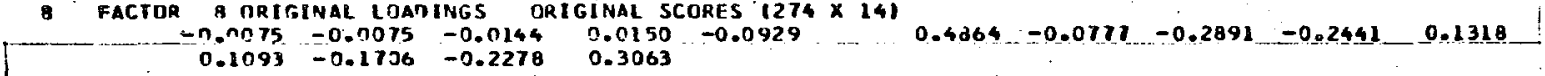

- Factor 9 original loajings ORIGINAL SCORES $(274 \times 14)$ $\begin{array}{llllllllll}-0.0145 & -0.0180 & 0.0497 & -0.2481 & -0.1473 & -0.0678 & 0.5460 & 0.0036 & -0.0156 & -0.0515\end{array}$ $\begin{array}{lllll}0.0863 & -0.2553 & 0.3564 & 0.2168\end{array}$

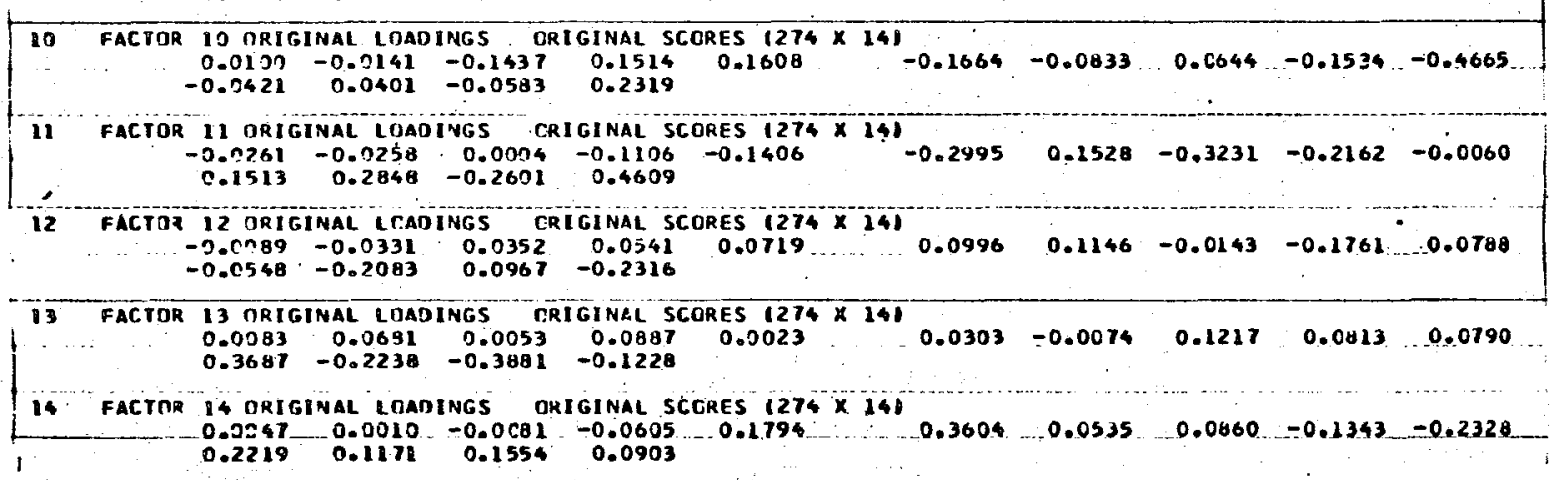


TABLE_28

ORIGINAL (PRE) SCORES AND POST SCORES - TYPICNL STUDX

COEFFICIENT OF INVARIANCE $\left(\mathrm{Z}^{*}\right.$

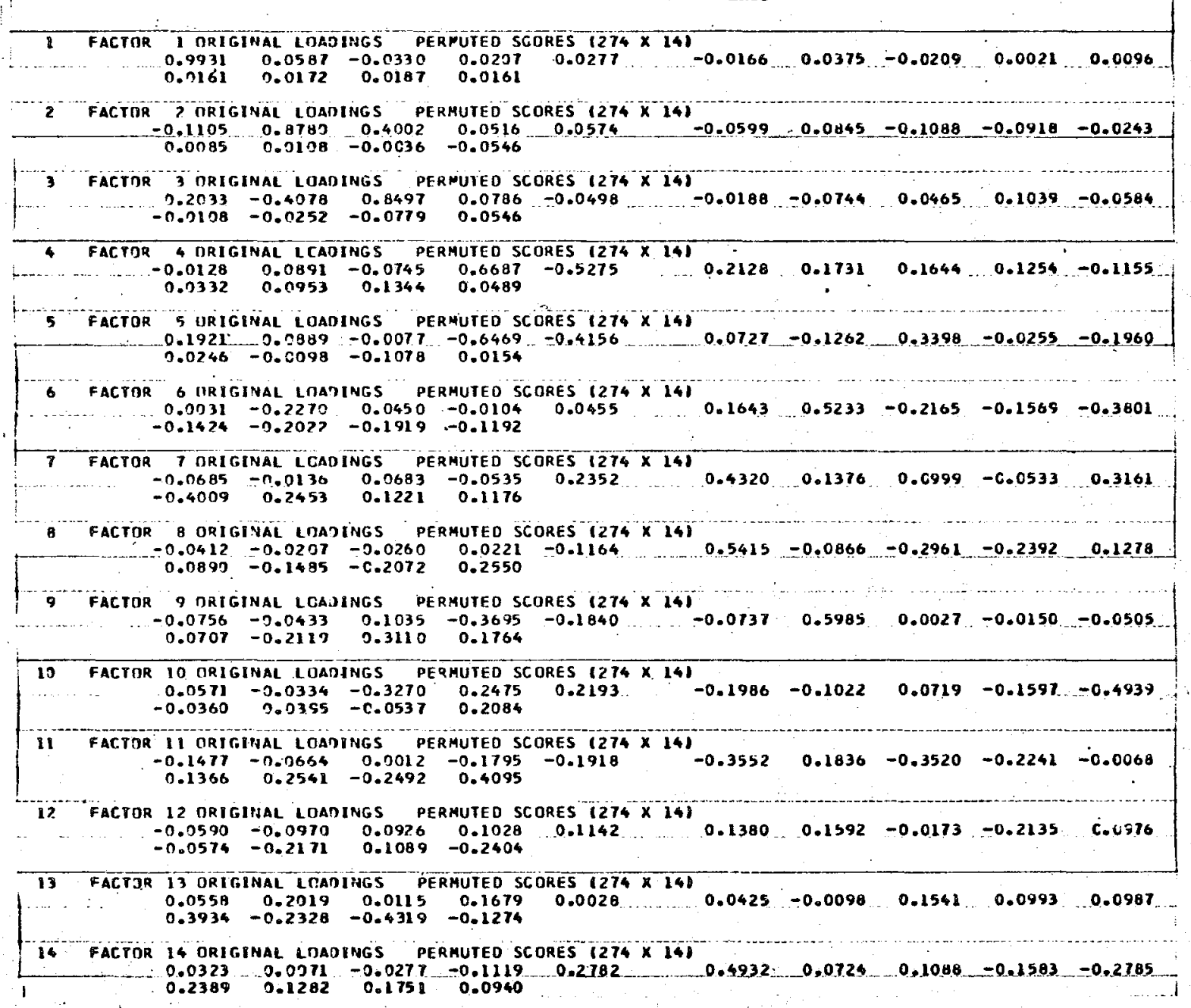


TABLE 29.

ORIGINAL (PRE) SCORES AND POST SCORES - TYPICAL STURY MODIFIED COEFFICIENT OF INVARIANCE

4

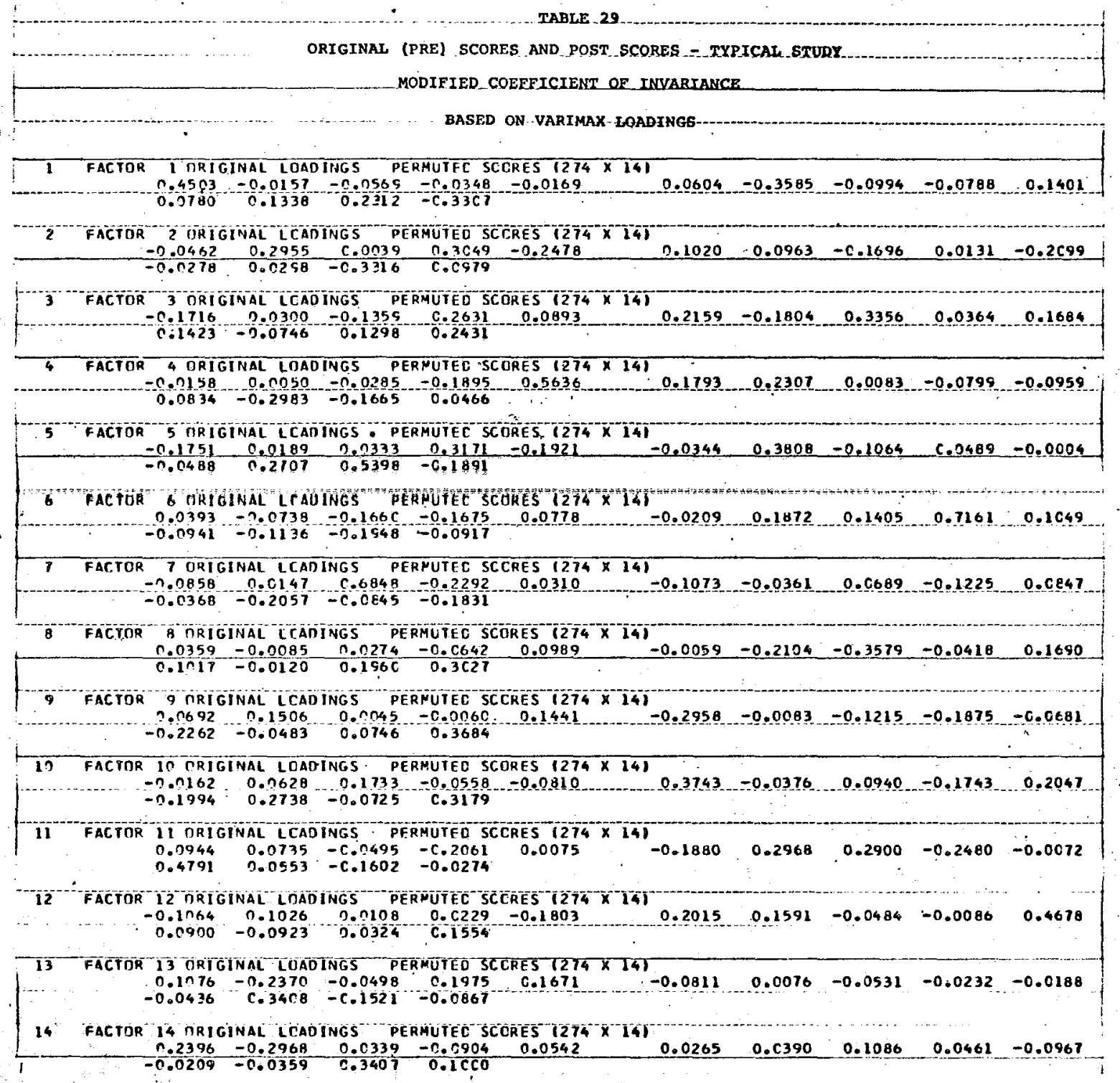


for the typical case. Factors I and I' and VII and VII' are highly related for this measure. Table 30 also indicates that the coefficients range from .0013 to .4985 .

Summary of Results for Typical Study

The results for factor scores based on principal components are as follows:

1. There is a larger number of highly related pre and post factors for the coefficient of invariance than for the coefficient of congruence.

2. In general, the numerical values are higher for the coefficient of invariance than for the coefficient of congruence.

3. The two solutions for the coefficlent of invariance are not the transpose of each other as was true for all five arbitrary cases.

The results for factor scores based on varimax loadings are as follows:

1. There is a larger number of highly related pre and post factors for the modified coefficient of invariance than for the coefficient of congruence.

2. With one exception, the magnitude of the relationship between factors which were highly related for both the modified coefficient of invariance and the coefficient of congruence was comparable. 
TABLE 30.

ORIGINAL (PRE) SCORES AND POST SCORES - TYPICAL STUDY

COEFFICIENT OF CONGRUENCE

BASED ON VARIMAX LOADINGS

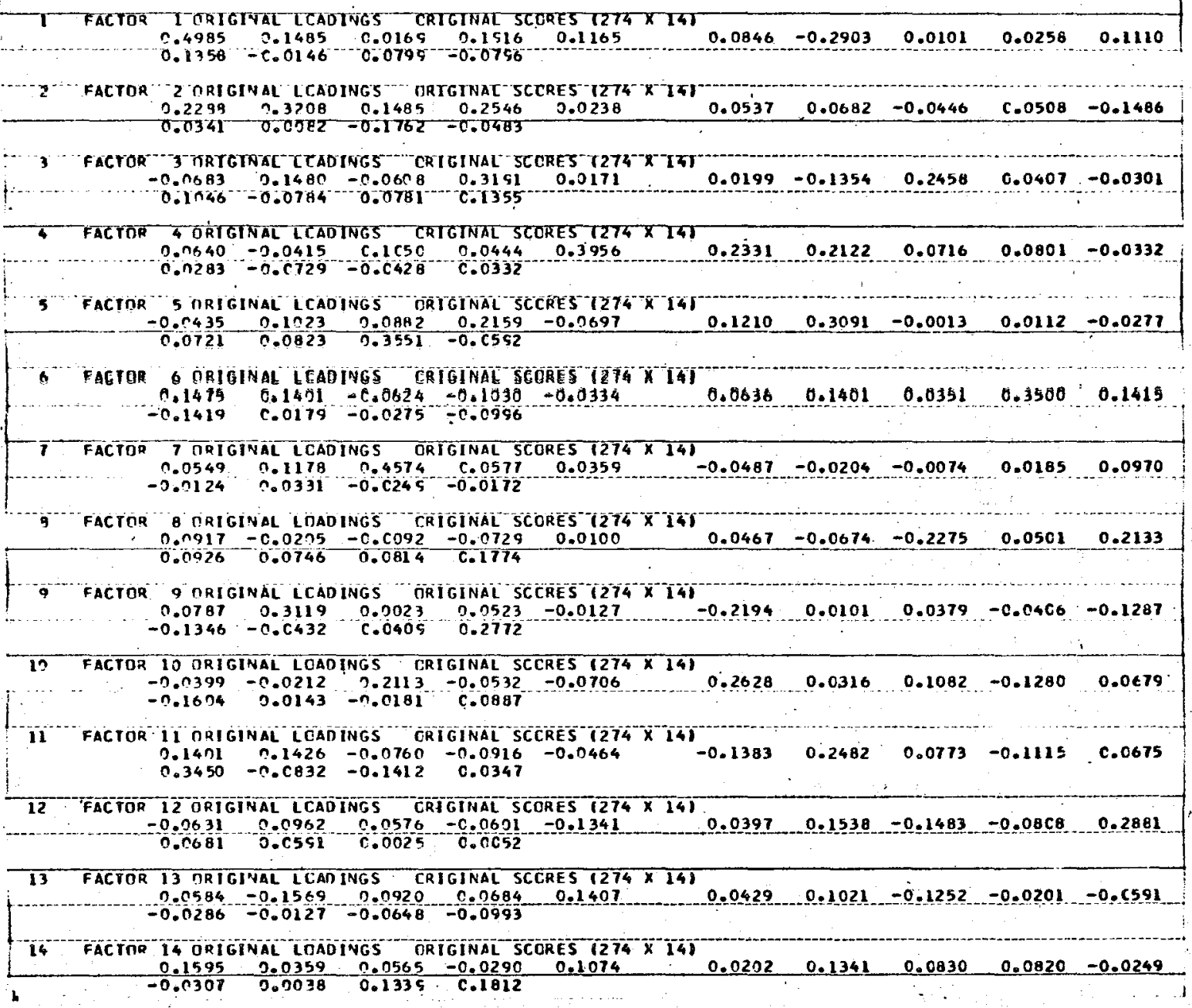




\section{Discussion}

This section considers implications of the results of

the three measures of invariance for the criteria specified. The findings reviewed also indicate the need for modification in the criteria for an adequate measure of invariance. In addition to their methodological significance, the implications of these results should be of value to investigators in selecting appropriate measures of invariance.

Independence of Factor Loadings

In the case of identical scores and procedures two sets of results were obtained. In the first of these the measures were obtained for factor scores based upon principal component factor loadings. In each instance the measure met the criteria of an adequate measure of invariance; i.e., an identity matrix. When the factor scores were based upon two sets of identical varimax factor loadings the values in the principal diagonal for all three measures were 1,0 indicating that the corresponding factors of the two matrices are in the same order and are perfectly related; however, only the coefficient of invariance and the coefficient of congruence produced an identity matrix. 
The modified coefficient of invariance based on identical varimax factor loadings showed a matrix with ones in the principal diagonal and non-zero correlations in the other cells. Since Pinneau, Schurr and Levine (1966) maintain that the modified coefficient is a direct reflection of the relationship among the loadings, question was raised concer ning the orthogonality of the varimax factors. Investiga! tion revealed that vectors for the set of varimax factors were not independent, i.e., $L^{\prime} I \neq D$, where $D$ is a diagonal matrix. Consequently, an adequate measure of the invariance of factors should reflect the relationships between different factors as well as the perfect correlation among the corresponding factors. The results of the coefficient of invariance and the coefficient of congruence incorrectly suggest that the set of varimax loadings is orthogonal, while the modified coefficient of invariance correctly indicates the lack of independence of vectors.

The issue of a non-orthogonal matrix of varimax loadings led to computation of the orthogonality of the : example of a varimax solution presented by Harman (1960); (Cf. Appendix, Table 32). The results confirmed the findings in this study, that the vectors are not orthogonal. Further calculations for the other varimax solutions also reveal that the factors obtained are not independent. Therefore, for each of the cases involving the varimax criteria, the modified coefficient of invariance can be expected to differ from the original 
criteria, as well as from the other measures of invariance, since it is the only measure which is sensitive to the lack of independence of the factor loadings.

Unique Solution for the Coefficient of Invariance

As mentioned earlier, there are two equations for the coefficient of invariance; one equation is based on $R_{11}$ and the other on $R_{22}$. The results for the artificial cases reveal that one set of coefficients is the transpose of the other. One solution can be shown to be equivalent to the other by reversing the order in which the factor scores are correlated:

$$
\begin{aligned}
R_{I V_{Z}} & =\frac{1}{\bar{N}} \mathrm{~S}^{* \prime} \mathrm{S} \\
& =\frac{1}{\bar{N}} \mathrm{~L}^{*-1} \mathrm{Z}^{\prime} \mathrm{Z} \mathrm{L}^{-1^{\prime}} \\
& =\mathrm{L}^{*-1} \mathrm{R}_{11} \mathrm{~L}^{-1^{\prime}} \\
& =\mathrm{L}^{*-1} \mathrm{~L} \cdot \mathrm{L}^{\prime} \mathrm{L}^{-1^{\prime}} \\
& =\mathrm{L}^{*-1} \mathrm{~L} \mathrm{I} \\
& =\mathrm{L}^{*-1} \mathrm{~L} .
\end{aligned}
$$

Equation 4, presented earlier, shows the formula for computing $\mathrm{R}_{I \mathrm{Z}_{\mathrm{Z}} \text { * }}$ without reversing the order of the factor scores, i.e.,

[4] $R_{I Z_{Z^{*}}}=\mathrm{L}^{-1} \mathrm{~L}^{*}$.

In the identical scores and procedures case $L^{*}=L$. If 
one substitutes $I$ for $L *$ in equations 4 and 10 one finds that

and

$$
\begin{aligned}
\mathrm{R}_{I V_{Z}} & =I^{-I} \mathrm{I} \\
\mathrm{R}_{I V_{Z^{*}}} & =I^{-I} \mathrm{I} \\
& =\mathrm{R}_{I V_{\mathrm{Z}}}
\end{aligned}
$$

In the case of permuting columns, $L^{*}=P \quad I$.

Substituting $P$ L for $L *$ in equations 4 and 10 one finds

$$
\mathrm{R}_{\mathrm{IV}}=\mathrm{L}^{-1} \mathrm{P}, \mathrm{L}
$$

and

$$
\begin{aligned}
\mathrm{R}_{\text {IVZ* }} & =\mathrm{L}^{-1} \quad \mathrm{P} \quad \mathrm{L} \\
& \equiv \mathrm{R}_{\mathrm{IVZ}} \cdot
\end{aligned}
$$

Thus the coefficient of invariance may be considered unique for the cases in which $\mathrm{z}^{*}$ is equivalent to $\mathrm{z}$ or $\mathrm{a}$ permutation of $\mathrm{Z}$.

In the typical study one solution for the coefficient of invariance is not the transpose of the other. The fact that the coefficient of invariance does not have a unique solution in the typical case is shown in the following. equations: $L *$ may not be replaced by either $L$ or $P$ the typical case, since the actual numerical loadings as well as their position in the new matrix change. Thus,

$$
\mathrm{R}_{I V_{Z}}=\mathrm{I*-1} \mathrm{L}
$$

and

$$
\mathrm{R}_{I V_{Z} *}=\mathrm{I}^{-1} \mathrm{~L}^{*}
$$

However, taking the inverse of $R_{I V_{Z}}$ yields 


$$
\begin{aligned}
\mathrm{R}_{I V_{Z}} & =\left(L^{*-1} L\right)^{-1} \\
& =L^{-1} L^{*}
\end{aligned}
$$

which is equal to the equation presented for $R_{I V_{Z^{*}}}$. While the coefficient of invariance does not have a unique solution in the typical case, one finds that one matrix is the inverse of the other. Hence an investigator who relies upon the coefficient of invariance must be prepared to either develop a rationale to employ one or the other equation, or compute both solutions and somehow resolve the discrepancies between them.

Row and Column Permutation

In the case of permuting rows and columns two unequal sets of factor loadings are obtained. Since $L \neq$, L* it is expected that an adquate measure of invariance would not result in an identity matrix; in addition as more rows and columns are permuted the relationship between the factors should decrease as more rows and columns were permuted. Empirical results for all three measures of invariance met the criterion; that is, none of the measures appear to satisfy the criteria extablished for an adequate measure of invariance in this case.

A comparison of the matrices obtained for permuted rows and columns with the results of permuting either rows or columns led to these findings:

a. The coefficient of congruence produced the same 
results for this case as it did for row permutation.

b. The coefficient of invariance and modified coefficient of invariance showed the same results for this i case as for column permutation.

Coefficient of Congruence. Since permuting rows does not modify $L_{,} L^{*}=L_{0}$ By substituting $P \quad Z$ for $Z^{*}$ in equation. 1, the equation for the coefficient of congruence . when rows of $\mathrm{Z}$ are permuted becomes

$$
\begin{aligned}
R_{C} & =\frac{I}{N} L^{-1} Z^{\prime} Z^{*} L^{*-I^{\prime}} \\
& =\frac{1}{N} L^{-1} Z^{\prime} \quad \text { P } Z L^{-I^{\prime}} \\
& =L^{-1} R_{12} \quad L^{-I^{\prime}}
\end{aligned}
$$

Permuting rows and columns results in $\mathrm{Z}^{*}=\mathrm{P} \mathrm{Z} \mathrm{P}^{*}$ and $L^{*}=P^{*} L_{0}$ By substitution, and recalling that $P=P^{\prime}$ $=\mathrm{P}^{-1}$

$$
\begin{aligned}
& R_{C}=\frac{1}{N} L^{-1} \quad Z^{\prime} \quad P \quad Z \quad P^{*} \quad P^{*} \quad L^{-I^{\prime}} \\
& =\frac{I}{N} \cdot L^{-1} \quad Z^{\prime} \quad P \quad Z \quad \text { I } \quad I \quad I^{-I^{\prime}} \\
& =\frac{1}{N} \quad L^{-1} \quad Z^{\prime} \quad P \quad Z \quad L^{-1} \\
& =\begin{array}{lll}
L^{-1} & R_{12} & I^{-1}
\end{array} .
\end{aligned}
$$

Thus the coefficient of congruence when rows are permuted is equal to the coefficient of congruence when rows and columns are permuted. Since the results of row and column permutation for this measure are the same values as those 
obtained when only the rows are permuted, the coefficient of congruence does not reflect changes in the factor loadings. Consequently, the coefficient of congruence does not in any way measure the invariance of the two sets of factor loadings.

Coefficient of Invariance. The following equations show that the coefficient of invariance for row and column permutation is equivalent to the coefficient of invariance when columns are permuted.

First consider the case of column permutation. Equations 7 and 8 , represented here, show the two solutions for the coefficient of invariance for this case; as was earlier shown one solution is the transpose of the other:

$$
\begin{aligned}
R_{I V_{Z}} & =L^{\prime} \quad P \quad L^{-1} \\
R_{I V_{Z^{*}}} & =L^{-1} \quad P \quad L \\
& =R_{I V_{Z}}^{\prime} .
\end{aligned}
$$

Now consider row and column permutation. When rows and columns of $\mathrm{Z}$ are permuted, $\mathrm{Z}^{*}=\mathrm{P}^{*} \mathrm{Z} \quad \mathrm{P}$ and $L^{*}=P$ L. Substituting for $L^{*}$ in equation 3 ,

$$
R_{I V}=L^{\prime} P L^{-1} \text {. }
$$

Thus, the first solution for this case is the same as equation. 7. The equation for the other solution involves $Z^{*}$, therefore, it is necessary to more completely develop the equations for $\mathrm{R}_{\mathrm{IV}} \mathrm{Z}^{*}$ :

$$
R_{I V^{*}}=\frac{I}{N} L^{-1} Z^{* I} Z^{*} L^{*-I^{\prime}}
$$


Substituting for $Z^{*}$ and $L *$,

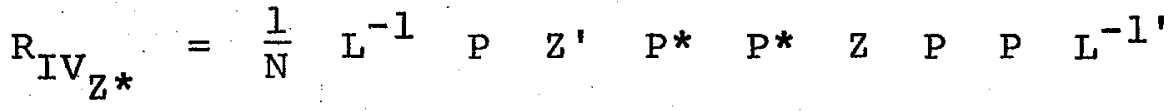

$$
\begin{aligned}
& =\frac{1}{\mathrm{~N}} \mathrm{~L}^{-1} \quad \mathrm{P} \quad \mathrm{Z}^{\prime} \quad \mathrm{I} \quad \mathrm{Z} \quad \mathrm{I} \quad \mathrm{L}^{-1^{\prime}} \\
& =\frac{1}{N} \cdot L^{-1} \quad P \quad Z^{\prime} \quad Z \quad L^{-I^{\prime}} \\
& =\mathrm{L}^{-1} \mathrm{P} \quad \mathrm{R}_{11} \quad \mathrm{~L}^{-1^{\prime}} \\
& =L^{-1} P \quad I \quad L^{\prime} . \quad L^{-1 !} \\
& =\mathrm{L}^{-1} \mathrm{P} \quad \mathrm{I} \quad \mathrm{I} \\
& =L^{-1} \mathrm{P} \quad \mathrm{L} \text {. }
\end{aligned}
$$

Comparing this equation with equation 8 shows that they are identical. Thus, equations for $R_{I V}$ and $R_{I V_{Z}}$ for row and column permutation are equivalent to $R_{I V}$ and $R_{I V}$ for column permutation.

Modified Coefficient of Invariance. Equation 9, presented earlier, shows the results of column permutation for this measure of invariance,

$$
\mathrm{R}_{\mathrm{IV}}^{0}=\mathrm{L}^{-1}, \mathrm{P} \quad \mathrm{L}^{-1^{\prime}}
$$

Permuting rows and columns results in the same equation as permuting columns since $\mathrm{Z}^{*}$ is not employed in the equation for the modified coefficient of invariance.

Summary and Conclusions. In summary, the coefficient of congruence yields the same results for row and column permutation as for row permutation. The coefficient of invariance and the modified coefficient of invariance reveal the same results for row and column permutation as 
for column permutation.

It was shown earlier that an adequate measure of invariance should reflect the fact that the factor loadings have remained identical in row permutation but they are modified by column permutation. The coefficient of congruence erroneously indicates that the factors are different when the rows are permuted. Since for this measure row and column permutation is equivalent to row permutation, the coefficient of congruence does not reflect the fact that the factor loadings are becoming more and more disparate.

Modified Coefficient of Invariance

In the case of identical scores and procedures based on varimax loadings the modified coefficient reflects the lack of independence of the factor loadings; this was discussed in the section "Independence of Factor Loadings." In this case the result did not agree with the original criterion of an adequate measure of invariance. However, as shown earlier, the original criteria should be modified so that it is applicable to non-orthogonal as well as orthogonal factors. Thus, the criteria for an adequate measure of invariance of factors should reflect the relationships between different factors as well as the perfect correlation among corresponding factors. Comparing the three measures of invariance for the case of identical scores and procedures based on varimax loadings 
reveals that only the modified coefficient of invariance meets the new criteria; i.e., this measure indicates that the two sets of varimax factor loadings are identical to each other but not orthogonal.

In the case of permuted rows the original criterion established for an adequate measure of invariance was an identity matrix. The modified coefficient did not conform to this criterion; however, the same modification in the criteria are applicable for this case as for the case of identical scores and procedures. The results of permuting rows were the same for $30 \%, 60 \%$ and $90 \%$ row permutation. In addition, these results were equal to those for identical scores and procedures based on varimax loadings. Thus, in each case of permuted rows the modified coefficient reflects the fact that the factors are not orthogonal as well as the fact that the factor loadings are identical. Since the values in the principal diagonal remain 1.0 for the row permutation case, the modified coefficient continues to accurately reflect the fact that permuting. rows of $\mathrm{z}$ does not change the resulting factor loadings.

Typical study

It will be recalled that when subjects' scores were interchanged (i.e., rows permuted) the coefficient of congruence failed to identify the fact that the factor loadings were identical. When variables were interchanged (i.e., columns permuted), the coefficient of congruence failed to identify the fact that the factor loadings were 
not identical. Thus, the coefficient of congruence does not measure the appropriate relationship between the two sets of factors under these conditions.

Pinneau and Newhouse (1964) contend that the coefficient of congruence confounds the relationship between the two sets of loadings by confusing the subject and variable differences. Indeed, the results of permuting rows and columns support this contention.

Comparing the center terms in the equations for the coefficient of congruence and the coefficient of invariance

$$
\begin{aligned}
R_{C} & =L^{-1} R_{12} L^{*^{-1}} \cdot \\
R_{I V Z} & =L^{-1} R_{11} L^{*-1} \cdot \\
R_{I V_{Z^{*}}} & =L^{-1} R_{22} L^{*-I^{\prime}}
\end{aligned}
$$

or

shows that the correlation matrices differ for these two measures. The matrix $\mathrm{R}_{12}$, employed by the coefficient of congruence, is comprised of both sets of standard scores, i.e., $\mathrm{R}_{12}=\frac{1}{\mathrm{~N}} \mathrm{Z}^{\prime} \mathrm{Z}^{*}$. The values which occur in this matrix are a function of the reliability of the variables and of the interval between pre and post test. The coefficient of invariance employs a correlation matrix $\left(R_{11}\right.$ or $\left.R_{22}\right)$ which is comprised of the intrarelationship among the variables obtained at the pre or post testing. Thus, the coefficient of invariance does not include a center term which employs divergent standard score matrices. The results in the typical study show that the 
coefficient of invariance reveals higher relationships between the factors than the coefficient of congruence. Since the coefficient of congruence has been shown to confound subjects and variables, the coefficient of invariance must be considered to be the more accurate measure of the relationships between the pre and post factors in the typical study.

The center term in the equation for the modified coefficient of invariance is a correlation matrix which is an identity matrix. Hence, the modified coefficients of invariance do not depend on off diagonal values.

Comparing the center terms for the modified coefficient of invariance and the coefficient of invariance shows that while the coefficient of invariance does not confound subjects and variables it does inflate the relationships between the factors by weighting the loadings by off diagonal values of the correlation matrix. The modified coefficient is a direct reflection of the comparability of the two sets of factor loadings since, by employing a correlation matrix which is an identity matrix, it does not modify the loadings. The results of 100\% row and column permutation support this conclusion: These results showed that while the factors were expected to have only chance relationships to each other, the coefficients of invariance were markedly higher than the modified coefficients of invariance.

The typical case illustrates another limitation of 
the coefficient of invariance. The five arbitrary cases yielded unique solutions for the coefficients of invariance or at least solutions which were the transpose of each other. In the typical study the coefficients of invariance are neither unique nor is one solution the transpose of the other. On the other hand, in the five arbitrary cases, as well as in the typical case, the modified coefficient of invariance does yield a unique solution. Thus, comparing the three measures of invariance for the typical study reveals that the modified coefficient of invariance is the only measure which directly reflects the comparability of the pre and post factor loadings.

\section{Conclusion}

Table 31 presents a summary of the analysis based on the original criteria. Since these criteria only apply for orthogonal factors, Table 31 indicates that the coefficient of invariance is the most adequate measure of invariance with the exception that it does not provide a unique solution in the typical case. When non-independent factors fare compared the coefficient of invariance erroneously considers these factors to be orthogonal. Changing the criteria such that an adequate measure of invariance should reflect both the similarity of corresponding factors as well as the relationship among different factors shows the modified coefficient of invariance to be the most adequate of the three measures compared. In addition, the 
results of the typical study, and the $100 \%$ row and column permutation case, indicate that the coefficient of invariance inflates the relationship between the factor loadings while the modified coefficient of invariance is a direct measure of this relationship. 
TABLE 31

A Comparison of Several Measures of Invariance

Summary of Analysis

\begin{tabular}{|c|c|c|c|c|}
\hline & $\begin{array}{l}\text { Predicted } \\
\text { Relationship }\end{array}$ & $\begin{array}{l}\text { nary of Analysis } \\
\text { Coefficient of } \\
\text { Congruence }\end{array}$ & $\begin{array}{l}\text { Coefficient of } \\
\text { Invariance }\end{array}$ & $\begin{array}{l}\text { Modified } \\
\text { Coefficient of } \\
\text { Invariance }\end{array}$ \\
\hline & & Re & $\mathrm{R}_{I V_{Z}} \quad \mathrm{R}_{I V_{Z^{*}}}$ & $\mathrm{R}_{I V}$ \\
\hline $\begin{array}{l}\text { Identical Scores } \\
\text { and Procedures (1) }\end{array}$ & $\frac{I}{(\text { Identity) }}$ & $I$ & I & I \\
\hline $\begin{array}{l}\text { Identical scores } \\
\text { and Rotation ( } 2)\end{array}$ & $\begin{array}{l}\text { T } \\
\text { (cosine of } \\
\text { angle of } \\
\text { separation) }\end{array}$ & $T$ & $\mathrm{~T}$ & $\neq \mathrm{T}$ \\
\hline $\begin{array}{l}\text { Permuted Scores- } \\
\text { Rows ( } 3 \text { ) }\end{array}$ & $\stackrel{I}{(\text { Identity })}$ & $<I$ & $I$ & $\neq I$ \\
\hline $\begin{array}{c}\text { Column Permutation } \\
\text { (4) }\end{array}$ & $\begin{array}{l}\text { Relationship } \\
\text { Will Decrease } \\
\text { or Identity }\end{array}$ & $I$ & $<I$ & $<I$ \\
\hline $\begin{array}{l}\text { Row and Column } \\
\text { Permutation (5) }\end{array}$ & $\begin{array}{l}\text { Relationships } \\
\text { Will Decrease } \\
\text { Same as Columns }\end{array}$ & $\begin{array}{l}<I \\
\text { Same as Rows }\end{array}$ & $\begin{array}{l}<\text { < } \\
\text { Same as columns }\end{array}$ & $\begin{array}{l}\text { < } I \\
\text { Same as columns }\end{array}$ \\
\hline Typical study (6) & & 、 & ${ }^{\text {Not Unique }}{ }^{\mathrm{IV}}=\stackrel{\mathrm{R}_{\mathrm{Z}}}{\mathrm{IV} \mathrm{Z}^{*}}$ & \\
\hline
\end{tabular}




\section{REFERENCES}

Harman, H. H. Modern Factor Analysis. Chicago: University of Chicago Press, 1960 .

Henrysson, S. Applicability of factor analysis in the behavioral sciences: A Methodological Study. Stockholm: Almqvist and Wiksell, 1957.

Kaiser, H. F. The varimax criterion for analytic rotation in factor analysis. Psychometrika, 1958, 23, 187-200.

Pinneau, S. R., and Newhouse, A. Measures of invariance and comparability in factor analysis for fixed variables. Psychometrika, 1964, 29, 271-281.

Pinneau, S. R., Schurr, B. S., and Levine, A. J. Coefficients of factor invariance and factor similarity for fixed variables. (in preparation) 1966.

Thomson, G. H. The Factorial Analysis of Human Ability. (5th ed.) Boston: Houghton Miffin $\overline{C o ., 1951 . ~}$

Thurstone, L. L. Multiple Factor Analysis. Chicago: University of Chicago Press, 1947.

Wrigley, C. S., and Neuhaus, J. O. The matching of two sets of factors. Amer. Psychologist, 1955, 10, 418-419. 
APPENDIX 
TABLE 32

"VARIMAX SOLUTION FOR EIGHT PHYSICAL VARIABLES"

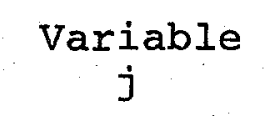

$$
\text { Initial solution }
$$

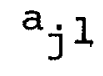

$a_{j}{ }^{2}$

Final Solution

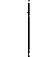

1

2

3

$$
4
$$

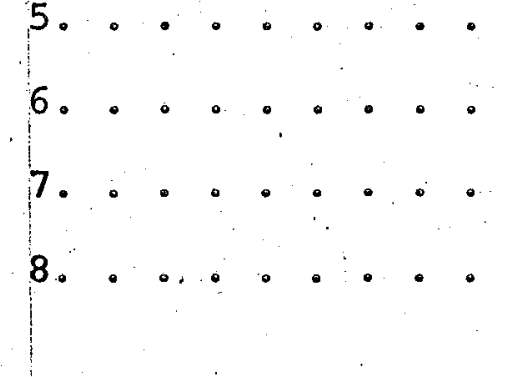

Sum of Crossproducts

.2175

$-.396 \quad .879$

$b_{j}{ }^{2}$

....... .830

$-.469^{\circ} \quad .919$

.272

2........ . .818

$-.470 \quad .890$

.210

3. . . . . . .777

$-.401 \quad .858$

.182

4. . . . . . . 798

$.500 \quad .238$

.246

.786

.458003

.900

$\therefore .672$

$.458 \quad .183$

.792

.594

$.444 \quad .135$

.729

.647

.333

.250

.684

Adapted from Harman (1960, p. 304-305) 